\title{
Homotopy coherent theorems of Dold-Kan type
}

\author{
Tashi Walde*
}

December 16, 2019

\begin{abstract}
We establish a large class of homotopy coherent Morita-equivalences of Dold-Kan type relating diagrams with values in any weakly idempotent complete additive $\infty$-category; the guiding example is an $\infty$-categorical Dold-Kan correspondence between the $\infty$-categories of simplicial objects and connective coherent chain complexes.

Our results generalize many known 1-categorical equivalences such as the classical DoldKan correspondence, Pirashvili's Dold-Kan type theorem for abelian $\Gamma$-groups and, more generally, the combinatorial categorical equivalences of Lack and Street.
\end{abstract}

\section{Contents:}

1 Introduction

1.1 Acknowledgements

\section{Preliminaries}

$2.1 \infty$-categorical notation and tools $\ldots \ldots \ldots \ldots$

2.2 Pointed $\infty$-categories . . . . . . . . . . . . . . . . . . .

2.3 Quotient categories and coherent chain complexes . . . . . . . . . . . . . 9

2.4 Additive and preadditive $\infty$-categories $\ldots \ldots \ldots \ldots \ldots \ldots$

2.5 Weakly idempotent complete $\infty$-categories . . . . . . . . . . . . . . . 11

3 The main theorem

3.1 DK-triples . . . . . . . . . . . . . . . . . . . . . 12

3.2 Key constructions . . . . . . . . . . . . . . . . . . . . 13

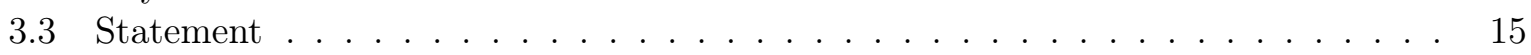

4 Examples 16

4.1 The $\infty$-categorical Dold-Kan correspondence ................. 16

4.2 Categories of partial maps . . . . . . . . . . . . . . . . . . . 18

5 The proof 20

5.1 Cofinality lemmas . . . . . . . . . . . . . . . . . 20

5.2 Inductive construction in the reduced case . . . . . . . . . . . . 22

5.3 Proof of the main theorem . . . . . . . . . . . . . . . 25

6 Comparison with... 26

6.1 ...the setting of Lack and Street . . . . . . . . . . . . . . . . . 26

6.2 ...Lurie's stable Dold-Kan correspondence . . . . . . . . . . . . . . . . 27

7 Application: measuring Kan extensions 28

References 30

${ }^{*}$ Rheinische Friedrich-Wilhelms-Universität Bonn, https://www .math.uni-bonn.de/people/twalde/ 


\section{Introduction}

The classical Dold-Kan correspondence [Dol58, Kan58] is a remarkable equivalence of categories

$$
\operatorname{Fun}\left(\Delta^{\mathrm{op}}, A\right) \stackrel{\simeq}{\longrightarrow} \mathrm{Ch}_{\geq 0}(A)
$$

between simplicial objects in $A$ and connective chain complexes in $A$, where $A$ is the category of abelian groups or, more generally, any abelian category [DP61]. In the past decades, many related equivalences have been constructed [Pir00, Sło04, Sło11, Hel14, CEF15, LS15] where the simplex category $\Delta$ is replaced by other categories which are of similar "combinatorial nature".

The goal of this article is to simultaneously generalize these equivalences in the homotopy coherent context of $\infty$-categories. To this end we study categories $B$ equipped with the structure $\mathrm{B}=\left(B, E, E^{\vee}\right)$ of a so-called DK-triple (see Definition 3.1.1); to each such DK-triple $\mathbb{B}$ we associate a pointed category $N_{0}=N_{0}(\mathrm{~B})$ and prove the following homotopy coherent correspondence of Dold-Kan type:

Theorem 1 (Corollary 3.3.4). For each weakly idempotent complet 1 ) additive 2 ) $\infty$-category $\mathcal{A}$, the DK-triple $\mathrm{B}$ induces a natura 3 ) equivalence

$$
\operatorname{Fun}(B, \mathcal{A}) \stackrel{\simeq}{\longrightarrow} \operatorname{Fun}^{0}\left(N_{0}, \mathcal{A}\right)
$$

between the $\infty$-categories of diagrams $B \rightarrow \mathcal{A}$ and of pointed diagrams $N_{0} \rightarrow \mathcal{A}$.

Before going into more details about DK-triples, we explain how Theorem 1 subsumes and generalizes previous results in the literature.

(1) In the case where $A$ is an abelian category, we recover the classical Dold-Kan correspondence (1.1) by applying Theorem 1 to $\mathcal{A}=A^{\text {op }}$ and to a suitable DK-triple $\mathbb{B}_{\Delta}=$ $\left(\Delta, E_{\Delta}, E_{\Delta}^{\vee}\right)$ whose associated pointed category $N_{0}\left(\mathbb{B}_{\Delta}\right)=\mathrm{Ch}_{\geq 0}$ is the shape of connective chain complexes; see Section 4.1 for more details.

(2) More generally, Theorem 1] specializes to the $\infty$-categorical Dold-Kan correspondence originally sketched by Joyal [Joy08, Section 35]

$$
\operatorname{Fun}\left(\Delta^{\mathrm{op}}, \mathcal{A}\right) \stackrel{\simeq}{\longrightarrow} \mathrm{Ch}_{\geq 0}(\mathcal{A})
$$

between simplicial objects and coherent connective chain complexes in any weakly idempotent complete additive $\infty$-category $\mathcal{A}$.

(3) Denote by $\mathbf{F i n}_{\star}$ the category of finite pointed sets and by Surj the category of (possibly empty) finite sets and surjections between them. Pirashvili [Pir00] constructed an equivalence

$$
\operatorname{Fun}\left(\operatorname{Fin}_{\star}, \mathbf{A b}\right) \stackrel{\simeq}{\longrightarrow} \operatorname{Fun}(\operatorname{Surj}, \mathbf{A b})
$$

between $\mathbf{F i n}_{\star}$-shaped and Surj-shaped diagram 4 ) of abelian groups. We recover this equivalence from Theorem 1 which more generally yields a natural equivalence

$$
\operatorname{Fun}\left(\operatorname{Fin}_{\star}, \mathcal{A}\right) \stackrel{\simeq}{\longrightarrow} \operatorname{Fun}(\operatorname{Surj}, \mathcal{A}),
$$

between $\Gamma$-object:5) and Surj-shaped diagrams in any weakly idempotent complete preadditive $\infty$-category $\mathcal{A}$; see Section 4.2 for more details.

\footnotetext{
1) weakly idempotent complete $=$ closed under direct complements

2) additive $=$ has direct sums and is enriched in abelian groups

3) natural in $\mathcal{A}$ with respect to additive functors

4) To be precise, Pirashvili only considers diagrams whose value on $\star \in \mathbf{F i n}_{\star}$ and on $\varnothing \in \mathbf{S u r j}$ is zero; these diagrams correspond to each other under the equivalence (1.3)

${ }^{5)}$ Some authors define $\Gamma$ to be the category $\mathbf{F i n}_{\star}$ of finite pointed sets; we use Segal's original definition $[\operatorname{Seg} 74]$ which is dual, i.e., $\Gamma:=\mathbf{F i n}_{\star}^{\text {op }}$. Regardless of the convention, a $\Gamma$-object in $\mathcal{A}$ is always a functor $\mathbf{F i n}_{\star} \rightarrow \mathcal{A}$.

6) preadditive $=$ has direct sums
} 
(4) Denote by FI $\sharp$ the category of finite sets and partial injections; let Fin $^{\simeq}$ be the groupoid of finite sets and bijections. For each commutative ground ring $R$, [CEF15, Theorem 4.1.5] (which is a special case of [Sło04, Theorem 1.5]) describes an equivalence

$$
\operatorname{Fun}(\mathrm{FI} \sharp, \operatorname{Mod}-R) \stackrel{\simeq}{\longrightarrow} \operatorname{Fun}\left(\mathbf{F i n}^{\simeq}, \operatorname{Mod}-R\right) \simeq \prod_{n \in \mathbb{N}}\left(\mathfrak{S}_{n}-\mathbf{R e p}_{R}\right)
$$

between the categories of FI\#-modules and of tuples of representations of all symmetric groups $\mathfrak{S}_{n}$. Again, our main result generalizes this equivalence to coherent diagrams/representations with values in arbitrary weakly idempotent complete preadditive $\infty$-categories.

(5) When $\mathcal{A}$ is an idempotent complete additive ordinary category, Theorem 1 recovers the general Dold-Kan type equivalence of Lack and Street [LS15, Theorem 6.8] which includes as special cases (1.1), (1.3), (1.5) and many more. See Section 6.1 for a detailed comparison.

(6) Some of the equivalences of Theorem 1 including the one for $\Gamma=\mathbf{F i n}_{\star}^{\text {op }}$ but not the one for $\Delta$-were already established by Helmstutler [Hel14] in the language of model categories; see Remark 4.2.3 for more details. Note that unlike Theorem 1, Helmstutler's result cannot be dualized so easily to yield, for instance, a model categorical version of the equivalence (1.4).

(7) In a stable $\infty$-category $\mathcal{D}$, coherent connective chain complexes can be encoded more conveniently as filtered objects, i.e., as diagrams $\mathbb{N} \rightarrow \mathcal{D}$; an explicit equivalence

$$
\operatorname{Fun}(\mathbb{N}, \mathcal{D}) \simeq \mathrm{Ch}_{\geq 0}(\mathcal{D})
$$

is part of Stefano Ariotta's Ph.D. thesis [Ari]. In this stable context, Lurie proved an $\infty$-categorical Dold-Kan correspondence [Lur17, Theorem 1.2.4.1] in the form of an equivalence

$$
\operatorname{Fun}\left(\Delta^{\mathrm{op}}, \mathcal{D}\right) \stackrel{\simeq}{\longrightarrow} \operatorname{Fun}(\mathbb{N}, \mathcal{D}),
$$

of $\infty$-categories; we expect this equivalence to agree with (1.1) under the identification (1.6). Note that while both equivalences (1.6) and (1.7) need the stability of $\mathcal{D}$ to work, Theorem 1 -just like the ordinary Dold-Kan correspondence - only needs that $\mathcal{A}$ is weakly idempotent complete additive. See Section 6.2 for a more detailled discussion.

We now introduce the notion of a DK-triple $\mathbb{B}=\left(B, E, E^{\vee}\right)$ on which Theorem 1 is based. It consists of a three-fold factorization system of type

$$
\bullet \stackrel{E}{\longrightarrow} \bullet \longrightarrow \stackrel{E^{\vee}}{\longrightarrow} \bullet,
$$

where the unnamed middle piece together with suitably encoded zero relations gives rise to the pointed category $N_{0}(\mathrm{~B})$ appearing on the right side of the equivalence (1.2). This notion is inspired by similar concepts appearing in [Sło04, Hel14, LS15]. We give an illustration in the examples of $\Gamma$ and $\Delta$, which are discussed in greater detail in Section 4.2 and Section 4.1.

- Every map $f: I \leftarrow J$ in $\Gamma=\mathbf{F i n}_{\star}^{\text {op }}$ can be written uniquely as the composition

$$
I \longleftarrow \operatorname{Im} f \longleftarrow \frac{J}{\operatorname{Ker} f} \longleftarrow J
$$

where

- the leftmost map is a bijection onto its image,

- the middle map is surjective and sends only the basepoint to the basepoint, in other words it just amounts to a surjection between the (possibly empty) sets obtained by omitting the basepoints,

- the rightmost map is bijective outside of its kernel (such maps are often called inert).

The category of those arrows of which appear as the middle piece of (1.8) is precisely (the opposite of) the category Surj; there are no zero relations in this case. 
- Every arrow in $\Delta$ can be written uniquely as the composition

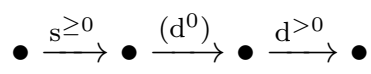

where

- the left arrow $\mathrm{s}^{\geq 0}$ is a (possibly empty) composition of codegeneracy maps,

- the middle arrow is either the identity or a 0-th coface map,

- the right arrow is a (possibly empty) composition of $i$-th coface maps $\mathrm{d}^{i}$ for $i>0$.

If one focuses only on the arrows of the second type, one obtains a category $\mathrm{Ch}_{\geq 0}$

$$
\underline{0} \underset{\mathrm{d}^{0}}{\longrightarrow} \underline{1} \underset{\cdots}{\stackrel{\mathrm{d}^{0}}{\longrightarrow}} \stackrel{2}{\stackrel{\mathrm{d}^{0}}{\longrightarrow} \cdots} \cdots
$$

with zero relations by declaring the composite of two 0-th coface maps to vanish (because it is not again a 0 -face map). Connective chain complexes are then exactly zero-preserving presheaves on $\mathrm{Ch}_{\geq 0}$. In order to properly encode the coherent zero relations in the $\infty$-categorical context, we actually consider $\mathrm{Ch}_{\geq 0}$ as a pointed category by adding an additional zero object through which all zero morphisms factor; for a more detailed explanation of this issue, see Section 2.3 .

\subsection{Acknowledgements}

This work was done during my Ph.D. studies at the Hausdorff Center for Mathematics (HCM); I am very grateful to my supervisors Catharina Stroppel and Tobias Dyckerhoff for supporting and encouraging me during this time. Moreover, I would like to thank Gustavo Jasso for many interesting discussions and for valuable feedback. This research was funded by the Deutsche Forschungsgemeinschaft (DFG, German Research Foundation) under Germany's Excellence Strategy - GZ 2047/1, Projekt-ID 390685813. This paper was written while the author was a guest at the Max Planck Institute for Mathematics (MPIM).

\section{Preliminaries}

\section{$2.1 \infty$-categorical notation and tools}

Throughout this article we use the language of $\infty$-categories/quasi-categories as developed by Joyal and Lurie; our main references are [Lur09] and Cis19].

We view the theory of $\infty$-categories as an extension of ordinary category theory by identifying an ordinary category $C$ with its nerve $\mathrm{N}(C)$. Typically, we use ordinary capital letters (e.g., $C, D, P, A)$ for 1-categories and the corresponding Euler Script letters (e.g., $\mathcal{C}, \mathcal{D}, \mathcal{P}, \mathcal{A})$ for $\infty$-categories.

We write $\operatorname{Fun}\left(\mathcal{C}^{\prime}, \mathcal{C}\right)$ for the $\infty$-category of functors $\mathcal{C}^{\prime} \rightarrow \mathcal{C}$. Given a small category $D$ and an $\infty$-category $\mathcal{C}$ a diagram of shape $D$ with values in $\mathcal{C}$ is a functor $D \rightarrow \mathcal{C}$; a $\mathcal{C}$-valued presheaf on $D$ is a functor $D^{\text {op }} \rightarrow \mathcal{C}$.

Given a category $C$ and an object $c \in C$, we denote by $C_{/ c}$ the category of objects over $c$; its objects are arrows of the form $\bullet \rightarrow c$. Given a functor $C^{\prime} \rightarrow C$ of categories and an object $c \in C$, we denote by $C^{\prime}{ }_{c}$ the relative overcategory defined as the fiber product $C^{\prime} \times_{C} C_{/ c}$ (with the functor $C^{\prime} \rightarrow C$ left implicit). Dually, the symbols $C_{c /}$ and $C^{\prime}{ }_{c /}$ denote absolute and relative undercategories.

We denote by $\mathcal{C}^{\triangleright}:=\mathcal{C} \star\{+\infty\}$ and $\mathcal{C}^{\triangleleft}:=\{-\infty\} \star \mathcal{C}$ the $\infty$-categories obtained from the $\infty$-category $\mathcal{C}$ by adjoining a new terminal object $+\infty$ or initial object $-\infty$, respectively. We denote by $\pi_{0} \mathcal{C}$ the set of equivalence classes of objects (i.e., the 0 -truncation), by he the homotopy category (i.e., the 1-truncation) and by $\mathfrak{e} \simeq$ the groupoid core (obtained by discarding noninvertible arrows) of $\mathcal{C}$. 
The main tool of this article is the theory of $\infty$-categorical (co)limits and Kan extensions as developed in [Lur09, Chapter 4]. We recall briefly the following key statements, which are analogs from classical facts of ordinary category theory and will be used throughout this article without further mention:

- [Lur09, Definition 4.3.2.2] Right/left Kan extension along a fully faithful functor $C^{\prime} \hookrightarrow C$ can be computed and characterized pointwise at each $d \in C$ by the induced limit/colimit of shape $C_{d /}^{\prime}$ and $C^{\prime}{ }_{d}$, respectively.

- Lur09, Proposition 4.3.2.15] Restriction along a fully faithful functor $C^{\prime} \hookrightarrow C$ induces an equivalence of $\infty$-categories between the full subcategories of $\operatorname{Fun}(C, \mathcal{C})$ and $\operatorname{Fun}\left(C^{\prime}, \mathcal{C}\right)$ consisting of those functors which are a right/left Kan extension and those functors which have a right/left Kan extension, respectively.

- Lur09, Corollary 4.3.2.16, Proposition 4.3.2.17] If every functor $C^{\prime} \rightarrow \mathcal{C}$ admits a right/left Kan extension along the fully faithful functor $C^{\prime} \hookrightarrow C$ then there is a unique fully faithful right/left Kan extension functor $\operatorname{Fun}\left(C^{\prime}, \mathcal{C}\right) \rightarrow \operatorname{Fun}(C, \mathcal{C})$ which is right/left adjoint to the restriction functor; its essential image is spanned by those functors $C \rightarrow \mathcal{C}$ which are a right/left Kan extension along $C^{\prime} \hookrightarrow C$.

- Lur09, Proposition 4.1.3.1] A functor $D^{\prime} \rightarrow D$ between ordinary categories is homotopy termina 7) if and only if each undercategory $D_{d /}^{\prime}$ (for each $d \in D$ ) is weakly contractible, i.e., has contractible geometric realization $\left|\mathrm{N}\left(D_{d /}^{\prime}\right)\right|$. Dually $D^{\prime} \rightarrow D$ is homotopy initial if and only if each overcategory $D^{\prime} / d$ is weakly contractible.

- Lur09, Proposition 4.1.1.8] The limit/colimit of a $D$-shaped diagram $D \rightarrow \mathcal{C}$ can be computed after precomposing with any homotopy initial/terminal functor $D^{\prime} \rightarrow D$.

Finally, we remind the reader that a localization 8 of an $\infty$-category $\mathcal{C}$ at a class $W \subset \mathcal{C}$ of arrows is a functor $\mathcal{C} \rightarrow \mathcal{C}\left[W^{-1}\right]$ which is universal amongst all functors that send the arrows in $W$ to equivalences. More precisely, for each $\infty$-category $\mathcal{C}^{\prime}$ the restriction functor

$$
\operatorname{Fun}\left(\mathfrak{C}\left[W^{-1}\right], \mathcal{C}^{\prime}\right) \longrightarrow \operatorname{Fun}\left(\mathcal{C}, \mathfrak{C}^{\prime}\right)
$$

is fully faithful with essential image consisting of those functors $\mathcal{C} \rightarrow \mathcal{C}^{\prime}$ that send all arrows in $W$ to equivalences in $\mathcal{C}^{\prime}$ (see, for example [Cis19, Definition 7.1.2]. Such $\infty$-categorical localizations always exist and are essentially unique, see [Cis19, Proposition 7.1.3].

\subsection{Pointed $\infty$-categories}

Recall, that an $\infty$-category $\mathcal{P}$ is called pointed if it has a zero object, i.e., an object $0 \in \mathcal{P}$ which is both initial and terminal in $\mathcal{P}$. A functor $\mathcal{P}^{\prime} \rightarrow \mathcal{P}$ between pointed $\infty$-categories is called pointed if it sends one (equivalently, each) zero object of $\mathcal{P}^{\prime}$ to a zero object of $\mathcal{P}$. We denote by $\mathbf{C a t}_{\infty}^{\mathbf{0}}$ the $\infty$-category of (small) pointed $\infty$-categories and pointed functors between them; it comes equipped with a canonical forgetful functor

$$
\mathrm{Cat}_{\infty}^{\mathrm{0}} \longrightarrow \mathrm{Cat}_{\infty}
$$

Given two pointed $\infty$-categories $\mathcal{P}^{\prime}$ and $\mathcal{P}$, we denote by $\operatorname{Fun}^{0}\left(\mathcal{P}^{\prime}, \mathcal{P}\right) \subset \operatorname{Fun}\left(\mathcal{P}^{\prime}, \mathcal{P}\right)$ the full subcategory spanned by the pointed functors.

Construction 2.2.1 (Free pointed category). Let $C$ be an ordinary category. We define a pointed category $C_{+}$by freely adjoining a zero object to $C$. Explicitly, it is described as follows:

- The objects of $C_{+}$are the objects of $C$ plus an additional object 0 .

\footnotetext{
7) Joyal and Lurie would say cofinal which, confusingly, is the word Cisinski uses for the dual concept (what we call homotopy initial). We avoid this potential confusion by using the hopefully unambiguous terminology of Dugger Dug.

8) Here our terminology differs from Lurie's who uses the word "localization" to refer to a special kind of localization functor which admits a fully faithful right adjoint (see Lur09, Definition 5.2.7.2 and Warning 5.2.7.3]).
} 
- For every object $x \in C_{+}$we put

$$
C_{+}(x, 0)=\{0\} \quad \text { and } \quad C_{+}(0, x)=\{0\}
$$

(in other words, $0 \in C_{+}$is a zero object as the notation suggests). Given objects $x, y \in C$, we set

$$
C_{+}(x, y):=C(x, y) \dot{\cup}\{0\}
$$

where here 0 denotes the composite map $x \rightarrow 0 \rightarrow y$.

- The composition in $C_{+}$is inherited from the composition in $C$.

The pointed category $C_{+}$comes equipped with the canonical (non-full) inclusion functor $C \rightarrow C_{+}$.

Construction 2.2.2 (Free pointed $\infty$-category). Let $\mathcal{C}$ be an $\infty$-category. Denote by

$$
\mathcal{C}^{\triangleleft \triangleright}:=\{-\infty\} \star \mathcal{C} \star\{+\infty\}
$$

the $\infty$-category obtained from $\mathcal{C}$ by freely adjoining an initial object $-\infty$ and a terminal object $+\infty$. We define $\mathcal{C}_{+}$to be the localization of $\mathcal{C}^{\triangleleft \triangleright}$ at the (essentially unique) edge $-\infty \rightarrow+\infty$ connecting the initial to the terminal object. The $\infty$-category $\mathcal{C}_{+}$is pointed (since localizations preserve both initial and terminal object:9) ) and comes equipped with the defining functor $\mathcal{C} \hookrightarrow$ $\mathrm{C}^{\triangleleft \triangleright} \rightarrow \mathcal{C}_{+}$.

If the category $\mathcal{C}$ in Construction 2.2.2 happens to be an ordinary category, then $\mathcal{C}^{\triangleleft \triangleright}$ is again an ordinary category. It is however not clear a priori that the the same is true for $\mathcal{C}_{+}$, because the localization procedure has the potential to turn an ordinary category into one that isn't. The following lemma addresses this issue.

Lemma 2.2.3. Let $C$ be an ordinary category. Then the functor $C \rightarrow C_{+}$from Construction 2.2.2 agrees with the one from Construction 2.2.1 In particular, $C_{+}$is an ordinary category again.

Proof. Let $C_{+}$be as in Construction 2.2.1 and consider the canonical functor

$$
\gamma: C^{\triangleleft \triangleright}=\{-\infty\} \star C \star\{+\infty\} \longrightarrow C_{+}
$$

given by the canonical inclusion of $C$ and by $-\infty,+\infty \mapsto 0$. We need to show that $\gamma$ exhibits $C_{+}$ as the $\infty$-categorical localization of $\{-\infty\} \star C \star\{+\infty\}$ at the unique map $-\infty \rightarrow+\infty$. Denote by $\langle-\infty,+\infty\rangle$ the full subcategory of $C^{\triangleleft \triangleright}$ spanned by $-\infty$ and $+\infty$. Since $\langle-\infty,+\infty\rangle \cong \Delta^{1}$ is weakly contractible, it follows by comparing universal properties that the desired localization can be computed as the pushout $C^{\triangleleft \triangleright} \sqcup_{\langle-\infty,+\infty\rangle}\{0\}$ (of $\infty$-categories). To conclude the proof, it therefore suffices to show that - after passing to nerves - the canonical square

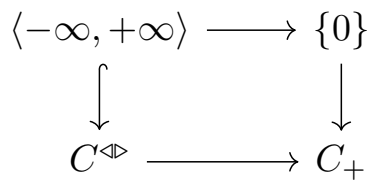

of categories becomes a (Joyal) homotopy pushout of simplicial sets. Since the left vertical map is a monomorphism, it suffices to show that the map

$$
\mathrm{N}(\{0\}) \sqcup_{\mathrm{N}(\langle-\infty,+\infty\rangle)} \mathrm{N}\left(C^{\triangleleft \triangleright}\right) \longrightarrow \mathrm{N}\left(C_{+}\right)
$$

from the (strict) pushout of simplicial sets is a (Joyal) weak equivalence; we will now show that it is in fact an inner anodyne extension.

The simplices of $C_{+}$can be described explicitly as follows: Each $m$-simplex of $\mathrm{N}\left(C_{+}\right)$is of the form

$$
\sigma(k, x, t): 0^{t(0)} \rightarrow x^{1} \rightarrow 0^{t(1)} \rightarrow x^{2} \rightarrow 0^{t(2)} \rightarrow \cdots \rightarrow 0^{t(k-1)} \rightarrow x^{k} \rightarrow 0^{t(k)},
$$

where

\footnotetext{
9) This follows, for instance, from Proposition 7.1.10 in Cis19]
} 
- $k$ is a natural number

- each $x^{i}: x_{0}^{i} \rightarrow \cdots \rightarrow x_{n(i)}^{i}($ for $1 \leq i \leq k)$ is an $n(i)$-simplex of $\mathrm{N}(C)$.

- $t(0), \ldots, t(k)$ are natural numbers of which all but $t(0)$ and $t(k)$ are required to be positive.

- $0^{t(i)}$ denotes a chain $0 \rightarrow \cdots \rightarrow 0$ with $t(i)$ many zeros.

- the dimension $m:=t(0)-1+\sum_{i=1}^{k}(n(i)+1+t(i))$ is non-negative.

Denote by $\mathrm{N}\left(C_{+}\right)^{\leq d} \subset \mathrm{N}\left(C_{+}\right)$the simplicial subset containing those simplices $\sigma(k, x, t)$ with $k \leq d$. The following are straightforward to verify:

(1) The map (2.1) induces an isomorphism $\mathrm{N}(\{0\}) \sqcup_{\mathrm{N}(\langle-\infty,+\infty\rangle)} \mathrm{N}\left(C^{\triangleleft \triangleright)} \cong \mathrm{N}\left(C_{+}\right)^{\leq 1}\right.$.

(2) For each $d \geq 1$, we have a pushout of simplicial sets

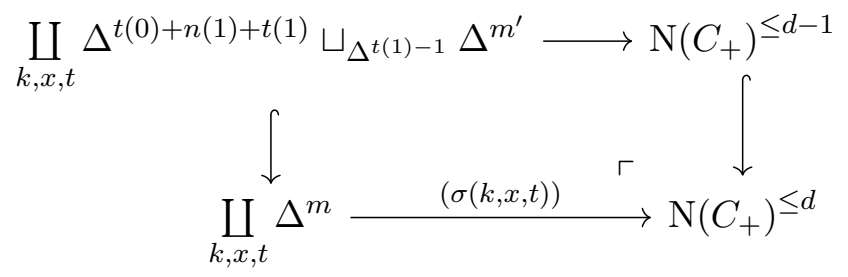

which corresponds to the decomposition of each chain (2.2) into the two overlapping chains

$$
0^{t(0)} \rightarrow x^{1} \rightarrow 0^{t(1)} \quad \text { and } \quad 0^{t(1)} \rightarrow x^{2} \rightarrow 0^{t(2)} \rightarrow \cdots \rightarrow 0^{t(k-1)} \rightarrow x^{k} \rightarrow 0^{t(k)}
$$

of dimensions $t(0)+n(1)+t(1)$ and $m^{\prime}:=-1+t(1)+\sum_{i=2}^{k}(n(i)+1+t(i))$, respectively.

(3) The simplicial set $\mathrm{N}\left(C_{+}\right)$is the union of the ascending chain $\mathrm{N}\left(C_{+}\right)^{\leq 1} \subset \mathrm{N}\left(C_{+}\right)^{\leq 2} \subset \cdots$ of simplicial subsets.

The left vertical map in the square (2.3) is an inner anodyne extension; it follows from (1), (2) and (3) that the same is true for the map (2.1); this concludes the proof.

Remark 2.2.4. In view of Lemma 2.2.3, we are justified in tacitly assuming that the free pointed $\infty$-category $C_{+}$on an ordinary category $C$ is given by the explicit description of Construction 2.2.1

The following lemma establishes the universal property of the free pointed $\infty$-category construction.

Proposition 2.2.5. Let $\mathcal{C}$ be a (small) $\infty$-category. For every pointed $\infty$-category $\mathcal{P}$, restriction along the functor $\mathrm{C} \rightarrow \mathrm{C}_{+}$induces an equivalence

$$
\operatorname{Fun}^{0}\left(\mathcal{C}_{+}, \mathcal{P}\right) \stackrel{\simeq}{\longrightarrow} \operatorname{Fun}(\mathcal{C}, \mathcal{P}) .
$$

of $\infty$-categories. In particular, the construction $\mathcal{C} \mapsto \mathcal{C}_{+}$yields a left adjoint to the forgetful functor $\mathbf{C a t}_{\infty}^{\mathbf{0}} \rightarrow \mathbf{C a t}_{\infty}$

Proof. The functors $\mathcal{C} \hookrightarrow \mathcal{C}^{\triangleleft} \hookrightarrow \mathcal{C}^{\triangleleft \triangleright} \longrightarrow \mathcal{C}_{+}$induce the following commutative diagram of 
functor $\infty$-categories and their various subcategories defined as indicated:

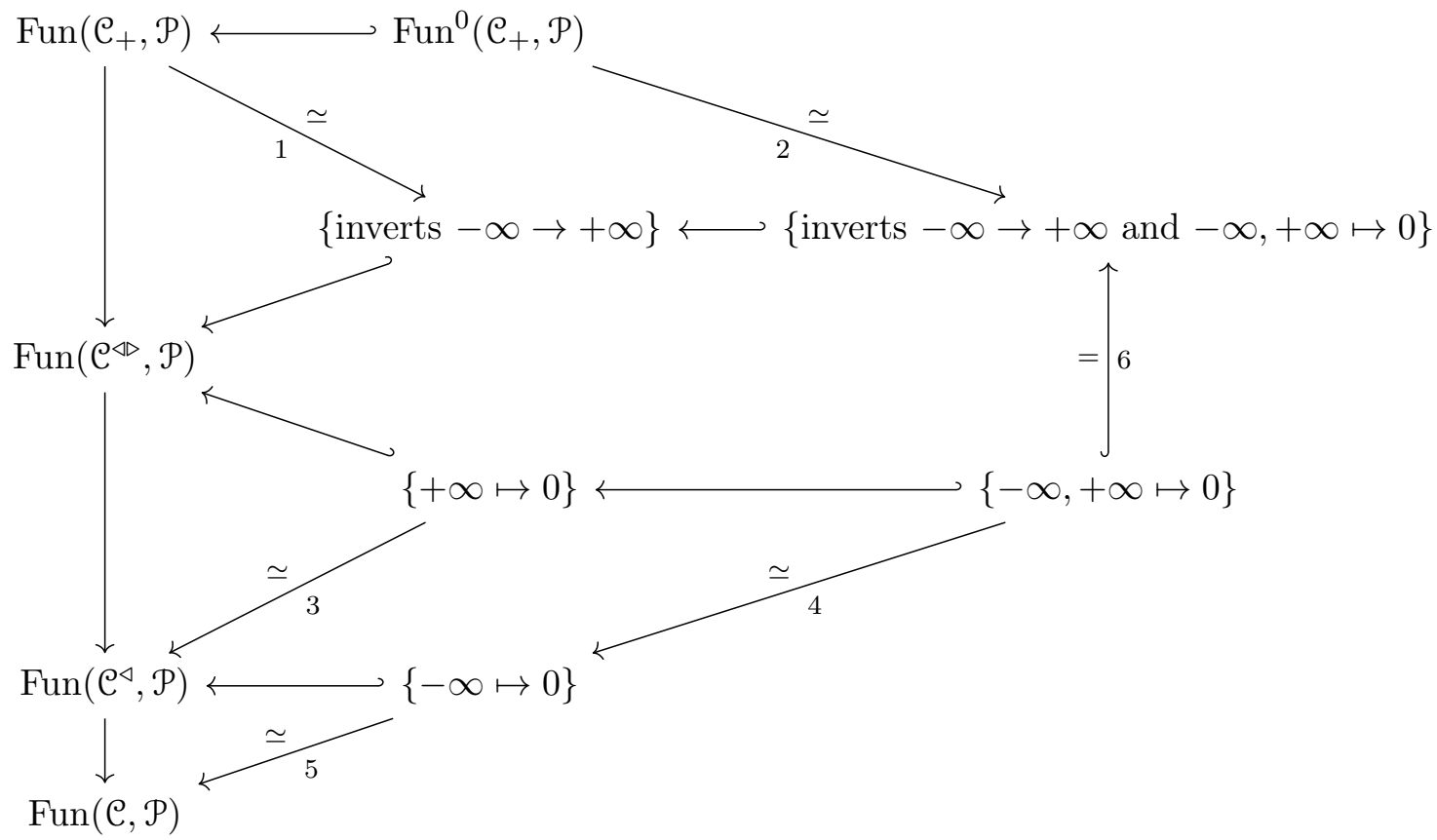

Restriction along $\mathcal{C}^{\triangleleft \triangleright} \rightarrow \mathcal{C}_{+}$induces the equivalence 1 by the universal property of the localization. The functors labeled by 3 and 5 are equivalences because they have an inverse given by right Kan extension and left Kan extension, respectively (using that $0 \in \mathcal{P}$ is a terminal and an initial object, respectively). The equivalences 2 and 4 are induced by restricting to appropriate full subcategories. Since $\operatorname{Map}_{\mathcal{P}}(0,0) \simeq$ pt, every functor $\mathcal{C}^{\triangleleft \triangleright} \rightarrow \mathcal{P}$ which sends $-\infty$ and $+\infty$ to zero objects must invert the edge $-\infty \rightarrow+\infty$; thus the inclusion labeled 6 is an equality of full subcategories. The result follows.

Lemma 2.2.6. Let $\mathcal{P}$ be a pointed $\infty$-category and let $\left\{f_{i}: X_{i} \rightarrow Y_{i} \mid i \in I\right\}$ be a finite set of morphisms in $\mathcal{P}$. Assume that the product

$$
\prod_{i \in I} f_{i}: \prod_{i \in I} X_{i} \longrightarrow \prod_{i \in I} Y_{i}
$$

exists in $\mathcal{P}$ and is an equivalence. Then for each $i \in I$, the morphism $f_{i}: X_{i} \rightarrow Y_{i}$ is an equivalence.

Proof. Given an inverse $g: \prod_{i} Y_{i} \rightarrow \prod_{i} X_{i}$ to $\prod f_{i}$, it is easy to see that for each $j \in I$ the composition

$$
Y_{j} \stackrel{\delta^{j}}{\longrightarrow} \prod_{i} Y_{i} \stackrel{g}{\longrightarrow} \prod_{i} X_{i} \stackrel{\pi_{j}}{\longrightarrow} X_{j}, \quad \text { where } \quad \pi_{i} \delta^{j}:= \begin{cases}\mathrm{Id}: Y_{j} \rightarrow Y_{j} & \text { if } i=j \\ 0: Y_{j} \rightarrow Y_{i} & \text { if } i \neq j\end{cases}
$$

is an inverse of $f_{j}$.

In a pointed $\infty$-category it makes sense to talk about fibers and cofibers which are the $\infty$-categorical analog of kernels and cokernels. The fiber and cofiber of an arrow $f: X \rightarrow Y$ are the pullback and pushout of the diagrams

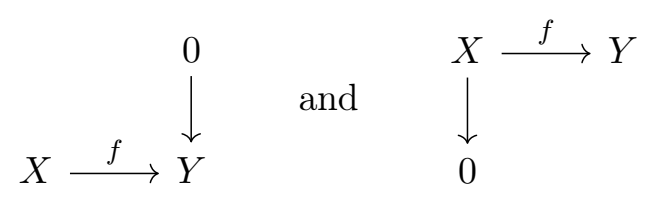

respectively. More generally, we define the total cofiber tot-fib $D$ of a conical diagram $D: K^{\triangleright} \rightarrow$ $\mathcal{P}$ as the cofiber of the canonical map $\operatorname{colim}\left(K \subset K^{\triangleright} \stackrel{D}{\rightarrow} \mathcal{P}\right) \rightarrow D(+\infty)$ and the total fiber 
tot-cof $D$ of a conical diagram $D: K^{\triangleleft} \rightarrow \mathcal{P}$ as the fiber of the canonical map $D(-\infty) \rightarrow \lim (K \subset$ $K^{\triangleleft} \stackrel{D}{\rightarrow} \mathcal{P}$ ). To recover the case of the ordinary fiber/cofiber set $K=\Delta^{0}$, hence $K^{\triangleright} \cong \Delta^{1} \cong K^{\triangleleft}$.

Another way of computing the total cofiber (resp. total fiber) of a $K^{\triangleright}$-shaped (resp. $K^{\triangleleft}$ shaped) diagram $D$ is to first pass to its right (resp. left) Kan extension along the first inclusion $K^{\triangleright} \hookrightarrow K^{\triangleright} \sqcup_{K} K^{\triangleright}$ (resp. $K^{\triangleleft} \hookrightarrow K^{\triangleleft} \sqcup_{K} K^{\triangleleft}$ )—which is given explicitly by setting the value on the cone point of the second copy of $K^{\triangleright}$ (resp. $K^{\triangleleft}$ ) to $0 \in \mathcal{P}$ - and then taking the colimit (resp. limit) of this diagram. The advantage of this description is that it is well defined even if the colimit (resp. limit) of $\left.D\right|_{K}$ does not exist in $\mathcal{P}$.

\subsection{Quotient categories and coherent chain complexes}

A chain complex in an ordinary pointed category $P$ is a diagram $\mathbb{Z}^{\mathrm{op}} \rightarrow P$, which we might depict as

$$
\ldots \stackrel{d}{\longleftarrow} \bullet \stackrel{d}{\longleftarrow} \bullet \stackrel{d}{\longleftarrow} \bullet \stackrel{d}{\longleftarrow} \ldots
$$

such that any composite of more than one $d$ is sent to the zero morphism in $P$. In other words, the category of chain complexes in $P$ is a full subcategory of the category $\operatorname{Fun}\left(\mathbb{Z}^{\mathrm{op}}, P\right)$ of $P$ valued presheaves on $\mathbb{Z}$. In the $\infty$-categorical world, this naive definition would no longer be satisfactory because

- for a map in an $\infty$-category, being zero is no longer a property but the structure of an explicit null-homotopy and

- there should be be higher coherence data exhibiting all the trivializations $d \circ \cdots \circ d \simeq 0$ as compatible

Let $C$ be a category equipped with an ideal $S \subseteq C$ (i.e., a set of arrows satisfying $C \circ S \circ C \subseteq S$ ), we would like to say what it means to equip a diagram $C \rightarrow \mathcal{P}$ with a coherent trivialization of all arrows in $S$.

Construction 2.3.1. We define a pointed category $\frac{C}{S}$ as follows:

- The objects of $\frac{C}{S}$ are the objects $x \in C$ plus an additional zero object 0 .

- The morphisms of $\frac{C}{S}$ are determined by setting

$$
\frac{C}{S}(x, y):=\frac{C(x, y)}{S} \cong\{f \in C(x, y) \mid f \notin S\} \dot{\cup}\{x \rightarrow 0 \rightarrow y\}
$$

for $x, y \in C$, with composition induced by the one in $C$.

The category $\frac{C}{S}$ comes equipped with the canonical functor $C \rightarrow \frac{C}{S}$ which is the identity on objects and sends precisely the arrows in $S$ to zero.

Remark 2.3.2. If $x \in C$ is an object with $\operatorname{Id}_{x} \in S$ then the unique morphisms $x \rightarrow 0$ and $0 \rightarrow x$ are mutually inverse isomorphisms in $\frac{C}{S}$.

Definition 2.3.3. Let $C \rightarrow \mathcal{P}$ be a $C$-shaped diagram in a pointed $\infty$-category $\mathcal{P}$. We say that a trivialization of all arrows in $S$ is an extension of $C \rightarrow \mathcal{P}$ along $C \rightarrow \frac{C}{S}$ to a pointed functor $\frac{C}{S} \rightarrow \mathcal{P}$.

Example 2.3.4. - The quotient $\frac{C}{\varnothing}$ of $C$ by the empty ideal is the free pointed category $C_{+}$. Hence Proposition 2.2.5 can be read as saying that the empty set of arrows can always be trivialized in a unique way.

- If the category $C$ is already pointed and $S=(0)$ consists of all zero maps $\bullet \rightarrow 0 \rightarrow \bullet$ then $\frac{C}{(0)} \cong C$.

- Every category $C$ has an ideal consisting of all non-isomorphisms; the corresponding quotient $\frac{C}{\nsucceq}$ is the free pointed category $C \widetilde{\simeq}$ on the groupoid core $C^{\simeq}$ of $C$. 
Definition 2.3.5. We denote by $\mathrm{Ch}:=\frac{\mathbb{Z}}{(\rightarrow \rightarrow)}$ the quotient of the poset $\mathbb{Z}$ by the ideal $(\rightarrow \rightarrow)$ of all maps $\underline{n} \rightarrow \underline{m}$ with $m-n \geq 2$. A coherent chain complex in $\mathcal{P}$ is a pointed presheaf $\mathrm{Ch}^{\text {op }} \rightarrow \mathcal{P}$; we denote by $\mathrm{Ch}(\mathcal{P}):=\operatorname{Fun}^{0}\left(\mathrm{Ch}^{\text {op }}, \mathcal{P}\right)$ the $\infty$-category of coherent chain complexes in $\mathcal{P}$. Similarly, we set $\mathrm{Ch}_{\geq 0}:=\frac{\mathbb{N}}{(\rightarrow \rightarrow)}$ and define the $\infty$-categories of connective chain complexes in $\mathcal{P}$ as $\mathrm{Ch}_{\geq 0}(\mathcal{P}):=\operatorname{Fun}^{0}\left(\mathrm{Ch}_{\geq 0}^{\mathrm{op}}, \mathcal{P}\right)$.

Remark 2.3.6. If $P$ is a pointed 1-category then it is straightforward to check that the restriction functor

$$
\operatorname{Fun}^{0}\left(\frac{C}{S}, P\right) \hookrightarrow \operatorname{Fun}(C, P)
$$

is fully faithful and that the essential image consists of those functors $C \rightarrow P$ which send arrows in $S$ to zero maps in $P$. This means that "sending arrows in $S$ to zero" is a property which a diagram $C \rightarrow P$ might or might not have. If $\mathcal{P}$ is an $\infty$-category, this is no longer true in general: specifying a lift of a diagram $C \rightarrow \mathcal{P}$ to a pointed diagram $\frac{C}{S} \rightarrow \mathcal{P}$ might require an infinite amount of additional structure.

Remark 2.3.7. Another way to make the notion of trivialization of arrows in $S$ precise would have been to work with $\infty$-categories enriched in pointed spaces or even in pairs of spaces. Then we could study pairs-enriched diagrams $C \rightarrow \mathcal{P}$, where $C$ is pairs-enriched via $S$ and where $\mathcal{P}$ is pairs-enriched (even $\mathcal{S}_{\star}$-enriched) via the zero maps. From this perspective one can see in a different way how the additional structure encoded in such trivializations comes in: unlike the forgetful functor Set $_{\star} \rightarrow$ Set from pointed sets to sets, the "forgetful" functor $\mathcal{S}_{\star} \rightarrow \mathcal{S}$ from the $\infty$-category of pointed spaces to the $\infty$-category of spaces is not faithful and in fact not even injective on $\pi_{0}$ of mapping spaces.

\subsection{Additive and preadditive $\infty$-categories}

Definition 2.4.1. GGN15, Definitions 2.1 and 2.6] An $\infty$-category $\mathcal{A}$ with finite products and coproducts is called preadditive if

- it is pointed, i.e., the canonical map $\varnothing \stackrel{\simeq}{\rightarrow} \star$ from the initial objects to the terminal object is an equivalence.

- for any two objects $X, X^{\prime} \in \mathcal{A}$, the canonical morphism

$$
\left(\begin{array}{cc}
\text { Id } & 0 \\
0 & \text { Id }
\end{array}\right): X \sqcup X^{\prime} \stackrel{\simeq}{\longrightarrow} X \times X^{\prime}
$$

(which exists, since $\mathcal{A}$ is pointed) is an equivalence.

The $\infty$-category $\mathcal{A}$ is called additive if additionally

- for each object $X \in \mathcal{A}$, the shear map

$$
\left(\begin{array}{cc}
\text { Id } & \text { Id } \\
0 & \text { Id }
\end{array}\right): X \sqcup X \stackrel{\simeq}{\longrightarrow} X \times X
$$

is an equivalence.

A functor between preadditive $\infty$-categories is called additive if it is pointed and preserves finite products (or, equivalently, finite coproducts).

Remark 2.4.2. Since products and coproducts in a preadditive $\infty$-category are canonically identified, it is customary to call them direct sums and denote them by the same symbol $\oplus$. $\diamond$

Remark 2.4.3. When specializing to the case where $\mathcal{A}$ is an ordinary category, Definition 2.4.1 recovers the classical notion of an additive category (as defined, for instance, in ML98, Chapter VIII]). However, we warn the reader that our use of the word "preadditive" (which is taken from [GGN15]) might be confusing, since many authors write "preadditive category" to mean a category enriched in abelian groups. 
Lemma 2.4.4. [Lur18, Definition C.1.5.1] Let $\mathcal{A}$ be an $\infty$-category with finite products and coproducts. Then $\mathcal{A}$ is preadditive/additive if and only if its homotopy category $\mathrm{h} \mathcal{A}$ is preadditive/additive.

Proof. The three maps defining the preadditivity/additivity of $\mathcal{A}$ in Definition 2.4.1 are sent by the functor $\mathcal{A} \rightarrow \mathrm{h} \mathcal{A}$ to the corresponding three maps defining the preadditivity/additivity of $\mathrm{h} \mathcal{A}$. Since a map is an equivalence in $\mathcal{A}$ if and only if it is an equivalence (i.e., isomorphism) in $\mathrm{h} \mathcal{A}$, the result follows.

Lemma 2.4.5. Let $\mathcal{A}$ be an additive $\infty$-category. Consider two $n$-tuples $\left(X_{i}\right)_{i=1}^{n},\left(Y_{i}\right)_{i=1}^{n}$ of objects of $\mathcal{A}$ and a matrix $F=\left(f_{i, j}: X_{i} \rightarrow Y_{j}\right)_{i, j=1}^{n}$ of maps between them. Assume that

- all diagonal entries $f_{i, i}: X_{i} \rightarrow Y_{i}$ are equivalences

- all entries below the diagonal (i.e., $f_{i, j}$ with $i>j$ ) factor through a zero object $0 \in \mathcal{A}$.

Then $F$, viewed as a map

$$
F: \coprod_{i=1}^{n} X_{i} \stackrel{\simeq}{\longrightarrow} \prod_{i=1}^{n} Y_{i},
$$

is an equivalence.

Proof. By passing to the homotopy category, we may reduce to the case of ordinary additive categories; Lemma 2.4.5 is standard in this case.

\subsection{Weakly idempotent complete $\infty$-categories}

Recall that an additive 1-category $A$ is called idempotent complete (or Karoubian) if every idempotent endomorphisms $p: X \rightarrow X$ induces a direct sum decomposition $X \cong \operatorname{Im} p \oplus \operatorname{Ker} p$. If $A$ is embedded as a full additive subcategory of some abelian category, this amounts to saying that $A$ is closed under summands, in particular, every abelian category is idempotent complete.

In the $\infty$-categorical world, the situation is a bit less favorable; for instance, even stable $\infty$-categories are not idempotent complete in genera 10). Fortunately for the purposes of this paper, the weaker condition of weak idempotent completeness will suffice. While idempotent completeness is a way to say that the category is "closed under summands", weakly idempotent completeness should be read as "closed under direct complements"; in other words $A$ is weakly idempotent complete additive if for each $X \in A$ and each direct sum decomposition $X \cong X^{\prime} \oplus X^{\prime \prime}$ (in some ambient abelian category) we have $X^{\prime} \in A$ if and only if $X^{\prime \prime} \in A$. One way to intrinsically make this definition without reference to any ambient category is to say that an additive category $A$ is weakly idempotent complete if each retraction (a.k.a. split epimorphism) has a kernel and each section (a.k.a. split monomorphism) has a cokernel (see for instance [TT90, A.5.1] and [Büh10, Definition 7.2]).

Next, we define weak idempotent completeness in the $\infty$-categorical setting. Let $\mathcal{P}$ be a pointed $\infty$-category. A section-retraction pair in $\mathcal{P}$, is a composable pair $(r, s)$ of maps in $\mathcal{P}$ whose composite $r \circ s$ is an equivalence. We say that two section-retraction pairs $(r, s)$ and $\left(r^{\prime}, s^{\prime}\right)$ are complementary, if they fit in a commutative diagram

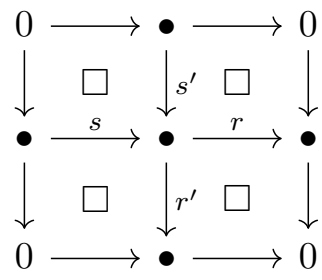

where all squares are biCartesian (i.e., both a pushout and a pullback); more precisely, we say that the diagram (2.4) exhibits $\left(r^{\prime}, s^{\prime}\right)$ as the complement of $(r, s)$, and vice versa.

\footnotetext{
10) Splitting a 1-categorical idempotent $p$ amounts to computing the kernels of $p$ and of Id $-p$ which exist in any abelian category. In contrast, the splitting a (coherent) $\infty$-categorical idempotent must be computed as an infinite limit which is not always possible.
} 
Remark 2.5.1. It is not hard to show using Kan extensions that the evident forgetful functor $\{$ diagrams $(2.4)$ in $\mathcal{P}\} \stackrel{\simeq}{\longrightarrow}$ section-retraction pairs in $\mathcal{P}$ which admit a complement $\}$

is an equivalence $\infty$-categories. This is the sense in which the complement of a section-retraction pair (together with the data exhibiting it as complementary) is essentially unique (if it exists).

Definition 2.5.2. A pointed $\infty$-category $\mathcal{P}$ is called weakly idempotent complete if every section-retraction pair has a complement.

Remark 2.5.3. When $\mathcal{P}=A$ is an additive 1-category, specifying a diagram (2.4) amounts to exhibiting $s^{\prime}$ as the kernel of $r$ and $r^{\prime}$ as the cokernel of $s$. Hence in this case Definition 2.5.2 agrees with the classical notion of weak idempotent completeness.

Example 2.5.4. Every stable $\infty$-category is weakly idempotent complete. More generally, each stable $\infty$-category gives rise to many examples by passing to subcategories which are closed under direct complements.

\section{The main theorem}

\subsection{DK-triples}

In this section we describe the axiomatic framework of DK-triples which encompasses - and is essentially equivalent - to the setting of Lack and Street [LS15]; see Section 6.1 for a detailed comparison. Similar ideas were already present in prior work of Słomińska [Sło04, Sło11] and of Helmstutler [Hel14] (cf. Remark 4.2.3).

Let $B$ be a category equipped with two subcategories $E, E^{\vee} \subset B$, each of which contains all isomorphism (in particular all objects). Arrows in $E$ and $E^{\vee}$ are called $E$ pis and dual $E$ pis, respectively; we depict them with the symbols $\rightarrow$ (a two-headed arrow) and $\longmapsto$ (a tailed arrow), respectively. For each $b \in B$ we denote by $E(b)$ the category of $E$ pis under 611 ; ; similarly, we denote by $E^{\vee}(b)$ the category of dual $E$ pis over $b$.

We make the following auxiliary definitions:

- We call an arrow in $B$ singular if it lies in the right ideal Sing $:=E_{\nsucceq}^{\vee} \circ B$ generated by the non-invertible dual Epis.

- An arrow which is not singular is called regular; we denote by Reg $:=B \backslash$ Sing the set of regular arrows.

- We call an arrow a Mono if it does not lie in the left ideal generated by the non-invertible Epis. We denote by $M:=B \backslash\left(B \circ E_{\nsucceq}\right)$ the set of Monos.

- For each $b \in B$ we have a pairing - o-: $E(b) \times E^{\vee}(b) \rightarrow \operatorname{Ar} B$ given by composition (where $\operatorname{Ar} B$ denotes the category of arrows in $B$ ). We denote by

$$
\langle-;-\rangle_{b}: \pi_{0} E(b) \times \pi_{0} E^{\vee}(b) \longrightarrow \pi_{0} \operatorname{Ar} B
$$

the induced pairing on isomorphism classes.

Definition 3.1.1. The datum $\mathbb{B}:=\left(B, E, E^{\vee}\right)$ is called

- A DK-triple 12 if it satisfies the following properties (using the auxiliary notation introduced above):

(T1) Every arrow $f$ of $B$ can be written uniquely (up to unique isomorphism) as a composition of the form

$$
\text { - } \stackrel{e^{\prime} \in E}{\longrightarrow} \bullet \stackrel{\bar{f} \in(M \cap \operatorname{Reg})}{\longrightarrow} \bullet \stackrel{e^{\vee} \in E^{\vee}}{\longrightarrow} \bullet
$$

\footnotetext{
11) The category $E(b)$ is nothing but the undercategory $E_{b}$ ( where $b$ is viewed as an object of $E$ ). We do not use the latter notation because it can unfortunately be confused with the undercategory $E_{b /}=B_{b /} \times_{B} E$ (where $b$ is viewed as an object of $B$ ).

12) Unsurprisingly, DK stands for Dold-Kan.
} 
(T2) For each $b \in B$, the pairing $\langle-;-\rangle_{b}$ can be described by a finite square matrix which is "unipotent upper triangular modulo non-isomorphisms",

i.e., there is a number $n \geq 1$ and bijections $\pi_{0} E(b) \cong\{1, \ldots, n\} \cong \pi_{0} E^{\vee}(b)$, such that the pairing $\langle-;-\rangle_{b}$ induces an $n \times n$-matrix

$$
\left(\begin{array}{cccc}
\simeq & ? & \cdots & ? \\
\neq & \ddots & \ddots & \vdots \\
\vdots & \ddots & \ddots & ? \\
\neq & \cdots & \neq & \simeq
\end{array}\right)
$$

with values in $\pi_{0} \operatorname{Ar} B$ which has invertible arrows on the diagonal and non-invertible arrows below the diagonal (there is no condition on the arrows above the diagonal).

(T3) The set $E^{\vee} \circ E$ is closed under composition.

(T4) The composition of two regular Monos is a (not necessarily regular) Mono, i.e., $(M \cap \operatorname{Reg}) \circ(M \cap \operatorname{Reg}) \subset M$

(T5) The singular arrows form a left module over $M$, i.e., we have $M \circ$ Sing $\subseteq$ Sing.

- a diagonalizable DK-triple if it if satisfies all axioms (T1) (T5) above and the matrix in (T2) can even be made diagonal modulo non-isomorphisms.

- reduced if $B=E^{\vee} \circ E$.

The following observations follow immediately from Definition 3.1.1.

Lemma 3.1.2. Let $\mathbb{B}=\left(B, E, E^{\vee}\right)$ be a DK-triple.

(1) For each $b$ there is a unique bijection $(-)^{\vee}: \pi_{0} E(b) \longleftrightarrow \pi_{0} E^{\vee}(b)$ such that for each $e \in E(b)$ the composition $e \circ e^{\vee}$ is an isomorphism in $B$.

(2) Every $E$ pi is a split epimorphism and every dual $E$ pi is a split monomorphism in $B$.

(3) For each $b \in B$, the categories $E(b)$ and $E^{\vee}(b)$ are both (equivalent to) posets.

(4) Both Reg and $M$ contain all isomorphisms of $B$.

(5) An arrow $B$ decomposed as in (3.1) is regular if and only if the component $e^{\vee} \in E^{\vee}$ is invertible and it is a Mono if and only if the component $e^{\prime} \in E$ is invertible.

(6) We have $M=(M \cap \operatorname{Reg}) \circ E$ and $\operatorname{Reg}=E^{\vee} \circ(M \cap \operatorname{Reg})$.

(7) The datum $\overline{\mathrm{B}}:=\left(\bar{B}, E, E^{\vee}\right)$ is again a DK-triple which is automatically reduced.

(8) If $\mathrm{B}$ is reduced then we have $M=E^{\vee}$ and $\operatorname{Reg}=E$ and $M \cap \operatorname{Reg}=B^{\simeq}$.

(9) If $\mathbb{B}$ is reduced then the dual datum $\mathbb{B}^{\mathrm{op}}:=\left(B^{\mathrm{op}},\left(E^{\vee}\right)^{\mathrm{op}}, E^{\mathrm{op}}\right)$ is again a (reduced) DKtriple.

Proof. Straightforward and left to the reader.

Each DK-triple $\mathbb{B}=\left(B, E, E^{\vee}\right)$ induces a canonical partial order $\leq$ on the set $\pi_{0} B$ by declaring $b^{\prime} \leq b$ if there exists a dual $E$ pi $b^{\prime} \longmapsto b$ or equivalently (by (1)] an $E$ pi $b \rightarrow b^{\prime}$. To see that $\leq$ is antisymmetric (i.e., $b \leq b^{\prime} \leq b$ implies $b \cong b^{\prime}$ ) choose an $E$ pi $e: b^{\prime} \rightarrow b$ and an $E$ pi $b \rightarrow b^{\prime}$ : the induced maps - o e: $\pi_{0} E(b) \hookrightarrow \pi_{0} E\left(b^{\prime}\right)$ and $\pi_{0} E\left(b^{\prime}\right) \hookrightarrow \pi_{0} E(b)$ are injective because $E$ pis are (split) epimorphisms. Since the sets $\pi_{0} E(b)$ and $\pi_{0} E\left(b^{\prime}\right)$ are finite by (T2), this implies that $e \circ-$ is a bijection; hence $e$ is a split monomorphism because $\operatorname{Id}_{b^{\prime}}$ lies in the image of $e \circ-$; hence $e$ is an isomorphism.

For each $b \in B$ the set $\left\{b^{\prime} \in \pi_{0} B \mid b^{\prime} \leq b\right\}$ of predecessors of $b$ is finite by (T2), hence the poset $\left(\pi_{0} B, \leq\right)$ is suited for inductive arguments.

\subsection{Key constructions}

Construction 3.2.1. Assume that $\mathbb{B}$ is a DK-triple. We define a pointed category $N_{0}=N_{0}(\mathbb{B})$ as the quotient

$$
N_{0}:=\frac{M}{M \cap \operatorname{Sing}}
$$

of $M$ by the two-sided ideal $M \cap$ Sing. Explicitly: 
- The pointed category $N_{0}$ has a zero object 0 and for each object $b \in B$ an object $\underline{b} \in N$

- For every pair of objects $\underline{b^{\prime}}, \underline{b} \in N$, we have the hom-set

$$
N_{0}\left(\underline{b}^{\prime}, \underline{b}\right):=\frac{M\left(b^{\prime}, b\right)}{(M \cap \operatorname{Sing})}=(M \cap \operatorname{Reg})\left(b^{\prime}, b\right) \dot{\cup}\left\{\underline{b^{\prime}} \rightarrow 0 \rightarrow \underline{b}\right\} .
$$

- Composition in $N_{0}$ is induced by composition in $B$; it is well defined because of (T4) and (T5).

For convenience we write $N$ for the full subcategory of $N_{0}$ spanned by all objects except the zero object 0 .

Remark 3.2.2. A particularly simple case of Construction 3.2.1 occurs when the set $M \cap$ Reg of regular Monos is closed under composition. In this case, $M \cap$ Reg is a subcategory of $B$ and the quotient $N_{0}:=\frac{M}{M \cap \operatorname{Sing}} \cong \frac{M \cap \operatorname{Reg}}{\varnothing} \cong \frac{N}{(0)}$ is simply the free pointed category on the category $M \cap$ Reg.

Notation 3.2.3. To minimize the potential confusion, we adopt the following conventions: Objects in $N$ are denoted by $n, n^{\prime}, n^{\prime \prime}$. Objects in $B$ are denoted by $b, b^{\prime}, b^{\prime \prime}$. Given an object $n \in N$, we denote by $[n]$ the corresponding object in $B$.

We now come to the key construction of this article.

Construction 3.2.4. Let $\mathbb{B}$ be a DK-triple. We define the pointed category $V=V(\mathbb{B})$ as the "upper triangular" category

$$
V:=\left(\begin{array}{cc}
N_{0} & R_{0} \\
0 & B_{+}
\end{array}\right):=\left(\begin{array}{cc}
\frac{M}{\operatorname{Sing}} & \operatorname{Sing} \backslash B \\
0 & B_{+}
\end{array}\right)
$$

associated to the $N_{0}-B_{+}$-bimodule $R_{0}:=\operatorname{Sing} \backslash B$. More precisely, the category $V$ is given explicitly as follows:

- The objects of $V$ are given by the objects $n \in N$, the objects $b \in B$ and a zero object 0 ; in other words we have $\mathrm{Ob} V:=\mathrm{Ob} N_{0} \sqcup_{\{0\}} \mathrm{Ob} B_{+}$.

- The hom-sets in $V$ between two objects of $N_{0}$ or between two objects of $B_{+}$are inherited from $N_{0}$ or from $B_{+}$, respectively.

- The only arrow in $V$ from an object $n \in N_{0}$ to an objects $b \in B_{+}$is the zero arrow $n \rightarrow 0 \rightarrow b$

- The set of arrows in $V$ from $b \in B$ to $n \in N$ is defined to be

$$
V(b, n):=R_{0}(b, n):=\operatorname{Sing} \backslash B(b,[n])=\operatorname{Reg}(b,[n]) \dot{\cup}\{b \rightarrow 0 \rightarrow n\}
$$

- Composition in $V$ is induced by the composition in $N_{0}$ and in $B_{+}$; the composition

$$
N_{0}\left(n, n^{\prime}\right) \times R_{0}(b, n) \times B_{+}\left(b^{\prime}, b\right) \longrightarrow R_{0}\left(b^{\prime}, n^{\prime}\right)
$$

is well defined because $M \circ$ Sing $\circ B \subseteq$ Sing.

The pointed category $V$ comes equipped with the two fully faithful embeddings

$$
B_{+} \longleftrightarrow V \longleftrightarrow N_{0} \text {; }
$$

for convenience we identify $B_{+}$and $N_{0}$ with their images in $V$.

Notation 3.2.5. We denote by $!:[n] \rightarrow n$ the arrow corresponding to the identity $\operatorname{Id}_{[n]} \in$ $\operatorname{Reg}([n],[n])$. For every non-zero arrow $u: b \rightarrow n$ in $V$ we denote by $[u] \in \operatorname{Reg}(b,[n])$ the corresponding regular arrow in $b$; in other words, $[u]: b \rightarrow[n]$ is the unique arrow satisfying $![u]=u$.

Remark 3.2.6. Assumptions (T4) and (T5) are needed to guarantee that Construction 3.2.1 and Construction 3.2.4 are well defined. In many examples $M$ is actually a subcategory of $B$; in this case $M \cap$ Sing is a two-sided ideal in $M$ in the usual sense and Construction 3.2.1 becomes an instance of Construction 2.3.1. The notation in Construction 3.2.1 and Construction 3.2.4 should be understood with this more special (but still very general) case in mind. 


\subsection{Statement}

We now state the main theorem of this article.

Theorem 3.3.1 (Homotopy coherent correspondences of Dold-Kan type). Let $\mathbb{B}=\left(B, E, E^{\vee}\right)$ be a DK-triple with associated pointed category $N_{0}=N_{0}(\mathrm{~B})$.

(a) For any weakly idempotent complete additive $\infty$-category $\mathcal{A}$, the restriction functors

$$
\text { Res: } \operatorname{Fun}^{0}(V, \mathcal{A}) \longrightarrow \operatorname{Fun}^{0}\left(B_{+}, \mathcal{A}\right) \quad \text { and } \quad \operatorname{Res}: \operatorname{Fun}^{0}(V, \mathcal{A}) \longrightarrow \operatorname{Fun}^{0}\left(N_{0}, \mathcal{A}\right)
$$

from Construction 3.2.4 admit a left adjoint LKE (left Kan extension) and a right adjoint RKE (right Kan extension), respectively.

(b) The composite adjunction

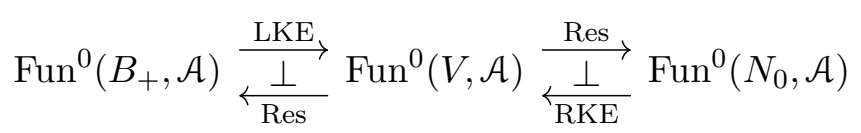

is an adjoint equivalence of $\infty$-categories.

(c) The adjoint equivalence (3.2) is natural in $\mathcal{A}$ with respect to additive functors.

(d) Consider a pointed functor $\mathcal{X}: B_{+} \rightarrow \mathcal{A}$ and denote by $\overline{\mathcal{X}}: N_{0} \rightarrow \mathcal{A}$ the pointed functor corresponding to $\mathcal{X}$ under the equivalence (3.2). Then for each $n \in N$ the canonical maps

$$
\underset{b \in E_{\nsucceq}^{\vee}([n])}{\operatorname{colim}} \mathcal{X}_{b} \longrightarrow \mathcal{X}_{[n]} \longrightarrow \lim _{b \in E_{\nsucceq}([n])} \mathcal{X}_{b}
$$

form a section-retraction pair with complement equivalent to $\overline{\mathcal{X}}_{n}$.

Remark 3.3.2. The notions of (pre)additivity and weak idempotent completeness are manifestly self-dual. Therefore in Theorem 3.3.1 (and all of the results below) we can replace the target $\infty$-category by its opposite, or, equivalently, $B_{+}$by $\left(B_{+}\right)^{\text {op }}$ and $N_{0}$ by $\left(N_{0}\right)^{\text {op }}$.

Remark 3.3.3. Since we are not assuming that our target category $\mathcal{A}$ has finite limits or colimits, it is not clear a priori that the limits/colimits indicated in (3.3) even exist; part of the statement of Theorem 3.3.1 (d) is that they do. Similarly, (a) is not automatic; in fact, the heart of the proof of Theorem 3.3.1 is an explicit inductive pointwise construction of the Kan extensions (3.2) in the case where $\mathbb{B}$ is reduced (see Proposition 5.2.1).

Corollary 3.3.4. In the situation of Theorem 3.3.1 the span $B \subset B_{+} \hookrightarrow V \hookleftarrow N_{0}$ induces a natural equivalence

$$
\operatorname{Fun}(B, \mathcal{A}) \stackrel{\simeq}{\longleftrightarrow} \operatorname{Fun}^{0}\left(N_{0}, \mathcal{A}\right)
$$

of $\infty$-categories for each weakly idempotent complete additive $\infty$-category $\mathcal{A}$.

Proof. Compose the equivalence of Theorem 3.3.1 with the natural equivalence

$$
\operatorname{Fun}(B, \mathcal{A}) \stackrel{\simeq}{\longleftarrow} \operatorname{Fun}^{0}\left(B_{+}, \mathcal{A}\right)
$$

produced by the universal property of the free pointed category $B \rightarrow B_{+}$.

Remark 3.3.5. In the situation of Remark 3.2.2, where $N_{0}=(M \cap \operatorname{Reg})_{+}$is a free pointed category, we can simplify the statement of Corollary 3.3.4 even more and obtain a natural equivalence

$$
\operatorname{Fun}(B, \mathcal{A}) \stackrel{\simeq}{\longrightarrow} \operatorname{Fun}(M \cap \operatorname{Reg}, \mathcal{A})
$$

between ordinary (i.e., non-pointed) $\infty$-categories of diagrams. All equivalences discussed in Section 4.2 are of this form. 
Specializing Corollary 3.3.4 to the 1-categorical case, we recover the main theorem of Lack and Street.

Corollary 3.3.6. [LS15, Theorem 6.8] Each DK-triple $\mathbb{B}=\left(B, E, E^{\vee}\right)$ induces a natural equivalence

$$
\operatorname{Fun}(B, A) \stackrel{\simeq}{\longrightarrow} \operatorname{Fun}^{0}\left(N_{0}(\mathbb{B}), A\right)
$$

of categories for each weakly 13$)$ idempotent complete additive category $A$.

Remark 3.3.7. Since the functor $\mathcal{A} \rightarrow \mathrm{h} \mathcal{A}$ to the homotopy category is additive, the naturality of equivalence (3.5) implies the existence of a commutative square

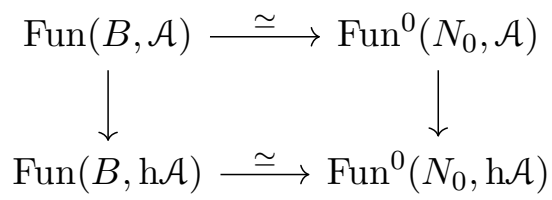

where the lower equivalence is an instance of Corollary 3.3.6.

Remark 3.3.8. If the DK-triple $\mathbb{B}$ is diagonalizable, then in all of the results above one can weaken the assumption on $\mathcal{A}$ and only require it to be weakly idempotent complete and preadditive. Indeed, the additivity of $\mathcal{A}$ is only used once (in the proof of Proposition 5.2.1) to invert certain upper triangular matrices in $\mathcal{A}$ obtained from the matrices $\langle-;-\rangle_{b}$ defined in $[\mathrm{T} 2)$ if $\mathbb{B}$ is diagonalizable then these matrices in $\mathcal{A}$ are diagonal, hence inverting them only requires preadditivity. See also Remark 5.2.4.

Remark 3.3.9. Theorem 3.3.1 (d) implies that one can compute the value $\overline{\mathcal{X}}_{n}$ of the diagram $\overline{\mathcal{X}}: N_{0} \rightarrow \mathcal{A}$ at an object $n \in N$ in two seemingly unrelated ways: as a total fiber

$$
\overline{\mathcal{X}}_{n} \simeq \text { tot-fib }(E([n]) \rightarrow B \stackrel{\mathcal{X}}{\rightarrow} \mathcal{A})
$$

along the Epis, or as a total cofiber

$$
\overline{\mathcal{X}}_{n} \simeq \text { tot-cof }\left(E^{\vee}([n]) \rightarrow B \stackrel{\mathcal{X}}{\rightarrow} \mathcal{A}\right)
$$

along the dual Epis.

\section{Examples}

\subsection{The $\infty$-categorical Dold-Kan correspondence}

We explain how to equip the simplex category $B=\Delta$ with the structure of a DK-triple; cf. [LS15, Example 3.2]. Recall that $\Delta$ is the category of finite non-empty linearly ordered sets and weakly monotone maps between them. We denote by $[n]$ the standard ordinal $\{0<1<\cdots<n\}$; every object of $\Delta$ is of this form up to unique isomorphism. Let $E \subset \Delta$ be the wide subcategory of surjective maps and let $E_{\min }^{\vee} \subset \Delta$ be the wide subcategory of those injectives maps that preserve minimal elements. The following observations are straightforward to verify and imply that $\mathrm{B}_{\min }^{\Delta}=\left(\Delta, E, E_{\min }^{\vee}\right)$ is a DK-triple:

- A map $f:[n] \rightarrow[m]$ is singular if and only if there is a non-minimal element of $[m]$ which is not in the image of $f$.

- The set $M$ of $M$ onos consists precisely of the injective maps in $\Delta$. Since $M$ is closed under composition, (T4) is satisfied.

13) To be precise, Lack and Street assume $A$ to be idempotent complete. 
- The set $M \cap$ Reg of regular monos consists of the identities and the 0-th coface maps

$$
\mathrm{d}^{0}:[n-1] \cong\{1<\cdots<n\} \longleftrightarrow[n] .
$$

Note that $M \cap$ Reg is not closed under composition.

(T1) Each map $[n] \rightarrow[m]$ in $\Delta$ admits a unique (up to unique isomorphism) factorization of type $E_{\min }^{\vee} \circ(M \cap \operatorname{Reg}) \circ E$, namely

$$
[n] \longrightarrow(\operatorname{Im} f) \longleftrightarrow(\{0\} \cup \operatorname{Im} f) \longmapsto[n] .
$$

(T3) The set $E_{\min }^{\vee} \circ E$ consists of the minimum-perserving arrows in $\Delta$, hence $E_{\min }^{\vee} \circ E$ is closed under composition.

(T5) If $0 \neq i \in[m]$ is a non-minimal element which is not in the image of $f:[n] \rightarrow[m]$ then, for each injective map $g:[m] \rightarrow\left[m^{\prime}\right]$, the element $0 \neq g(i) \in\left[m^{\prime}\right]$ is not minimal and not contained in the image of $g \circ f$.

(T2) For each $[n],[m] \in \Delta$ we have a bijection

$$
(-)^{\vee}: E([n],[m]) \stackrel{\cong}{\longrightarrow} E_{\min }^{\vee}([m],[n])
$$

which sends a surjection $e:[n] \rightarrow[m]$ to its minimal section $e^{\vee}:[n] \rightarrow[n]$ given by the formula $i \mapsto \min e^{-1}\{i\}$. A composition $e^{\prime} \circ e^{\vee}$ of an Epi $e^{\prime}:[n] \rightarrow[m]$ with a dual Epi $e^{\vee}:[n] \longmapsto[m]$ is

- an isomorphism if $e^{\prime}=e$

- not an isomorphism if $e^{\vee} \nsucceq e^{\prime \vee}$ poinwise as maps $[m] \rightarrow[n]$

(note that we make no claim when $e^{v}<e^{\vee}$ ). Hence, for each $[n] \in \Delta$, the lexicographic ordering on $\pi_{0} E_{\min }^{\vee}([n])$ makes the matrix

$$
\pi_{0} E_{\min }^{\vee}([n]) \times \pi_{0} E_{\min }^{\vee}([n]) \underset{(-)^{\vee}}{\cong} \pi_{0} E([n]) \times \pi_{0} E_{\min }^{\vee}([n]) \stackrel{\langle-;-\rangle_{[n]}}{\longrightarrow} \pi_{0} \operatorname{Ar} \Delta
$$

into upper triangular shape modulo non-isomorphisms.

Example 4.1.1. The matrix $\langle-;-\rangle_{[2]}$, can be depicted as follows

\begin{tabular}{c|cccc} 
& 0 & 01 & 02 & 012 \\
\hline$(012)$ & $\mathbf{0}$ & $(01)$ & $(12)$ & $(012)$ \\
$0(12)$ & 0 & 01 & 02 & $0(12)$ \\
$(01) 2$ & 0 & $(01)$ & $\mathbf{0 2}$ & $(01) 2$ \\
012 & 0 & 01 & 02 & $\mathbf{0 1 2}$
\end{tabular}

where the rows are labeled by equivalence classes of $E$ pis $[2] \rightarrow[m]$ (written by grouping elements with the same image); dually, the rows are labeled by equivalence classes of dual Epis $[m] \rightarrow[2]$ (written by listing the elements in the image). The isomorphisms are highlighted in red/bold, showing that the matrix is - modulo non-isomorphisms - unipotent upper triangular but not diagonal. In particular, this example shows that the DK-triple $\mathbb{B}_{\min }^{\Delta}$ is not diagonalizable. $\diamond$

There is an equivalence $\mathrm{Ch}_{\geq 0}^{\mathrm{op}}:=\frac{\mathbb{N}}{(\leftarrow \leftarrow)} \stackrel{\simeq}{\rightarrow} N_{0}:=\frac{M}{M \cap \operatorname{Reg}}$ of pointed categories which is given on objects by $\underline{n} \mapsto[n]$ and is determined on morphisms by sending the arrow $\underline{n} \rightarrow \underline{n+1}$ to the 0 -th coface map d $\mathrm{d}^{0}:[n] \rightarrow[n+1]$. Applying Corollary 3.3.4 to the DK-triple $\mathbb{B}_{\min }^{\bar{\Delta} \text { thus }}$ establishes a natural equivalence

$$
\operatorname{Fun}(\Delta, \mathcal{A}) \stackrel{\simeq}{\longrightarrow} \operatorname{Fun}^{0}\left(\mathrm{Ch}_{\geq 0}^{\mathrm{op}}, \mathcal{A}\right)=\mathrm{Ch}_{\geq 0}^{\mathrm{op}}(\mathcal{A})
$$

for each weakly idempotent complete additive $\infty$-category $\mathcal{A}$. Replacing $\mathcal{A}$ by its opposite (which is again weakly idempotent complete additive) yields the more familiar form of the following $\infty$-categorical Dold-Kan correspondence: 
Corollary 4.1.2. The DK-triple $\mathbb{B}_{\min }^{\Delta}=\left(\Delta, E, E_{\min }^{\vee}\right)$ induces a natural equivalence of $\infty$-categories

$$
\operatorname{Fun}\left(\Delta^{\mathrm{op}}, \mathcal{A}\right) \stackrel{\simeq}{\longrightarrow} \mathrm{Ch}_{\geq 0}(\mathcal{A})
$$

between simplicial objects and connective chain complexes in any weakly idempotent complete additive $\infty$-category $\mathcal{A}$.

The simplex category $\Delta$ is part of a second DK-triple $\mathbb{B}_{\max }^{\Delta}$, where $E$ is again the set of surjections and $E_{\max }^{\vee}$ is the set of maximum-preserving injections in $\Delta$. The DK-triples $\mathbb{B}_{\min }^{\Delta}$ and $\mathrm{B}_{\max }^{\Delta}$ have canonically isomorphic quotient categories $N_{0}\left(\mathbb{B}_{\text {min }}^{\Delta}\right) \cong \mathrm{Ch}_{\geq 0}^{\text {op }} \cong N_{0}\left(\mathbb{B}_{\max }^{\Delta}\right)$ and correspond to each other under the canonical involution $\uparrow: \Delta \stackrel{\cong}{\leftrightarrows} \Delta$ which reverses the linear order on each object of $\Delta$. Hence we have a commutative diagram

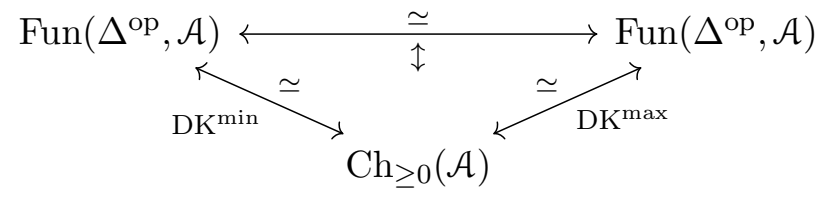

which intertwines the corresponding two versions of the Dold-Kan functor (4.1).

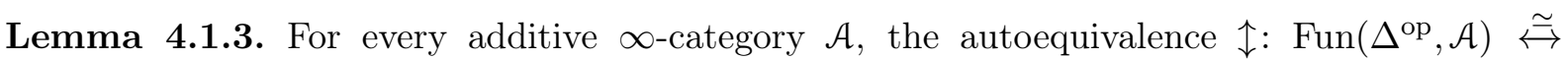
$\operatorname{Fun}\left(\Delta^{\mathrm{op}}, \mathcal{A}\right)$ is equivalent to the identity; in other words, the two Dold-Kan functors $\mathrm{DK}^{\mathrm{min}}$ and $\mathrm{DK}^{\max }$ agree (up to equivalence).

In the 1-categorical context, one can check explicitly that $\mathrm{DK}^{\mathrm{min}}$ and $\mathrm{DK}^{\mathrm{max}}$ both agree (up to natural isomorphism) with the normalized chain functor, hence with each other. For $\infty$-categories we provide the following alternative argument:

Proof. Choose a stable $\infty$-category $\mathcal{A} \subseteq \mathcal{D}$ into which $\mathcal{A}$ is embedded as a full additive subcategory; for instance the Yoneda embedding into the $\infty$-category of additive spectral presheaves $\mathcal{A}^{\text {op }} \rightarrow \mathcal{S} p$ (for more details, see for instance Appendix C.1.5 in [Lur18]). Since the involution $\uparrow: \Delta \stackrel{\cong}{\leftrightarrows} \Delta$ preserves the filtration

$$
\Delta_{\leq 0} \subset \Delta_{\leq 1} \subset \cdots \subset \Delta_{\leq n} \subset \cdots \subset \Delta,
$$

the functor $\operatorname{Fun}\left(\Delta^{\mathrm{op}}, \mathcal{D}\right) \rightarrow \operatorname{Fun}(\mathbb{N}, \mathcal{D})$, which sends a simplicial objects to its sequence of partial colimits, is $\uparrow$-invariant. Since $\mathcal{D}$ is stable, Lurie's stable Dold-Kan correspondence (which we also discuss in Section 6.2 states that this functor is an equivalence; hence the ( $\downarrow$-invariant) composition

$$
\operatorname{Fun}\left(\Delta^{\mathrm{op}}, \mathcal{A}\right) \hookrightarrow \operatorname{Fun}(\mathbb{N}, \mathcal{D}),
$$

is fully faithful. The result follows.

\subsection{Categories of partial maps}

An important class of examples of diagonalizable DK-triples arises by considering partial maps with respect to certain factorization systems; we sketch here the corresponding discussion by Lack and Street [LS15, Example 3.1]. Let $(\mathscr{E}, \mathscr{M})$ be a factorization system on a category $\mathscr{A}$, i.e.,

- $\mathscr{E}$ and $\mathscr{M}$ are subcategories of $\mathscr{A}$ containing all isomorphisms and

- arrows in $\mathscr{A}$ factor, uniquely up to unique isomorphism, as compositions of the type $\mathscr{M} \circ \mathscr{E}$; assume furthermore that

- the arrows in $\mathscr{M}$ are monomorphisms,

- each object of $\mathscr{A}$ has only finitely many $\mathscr{M}$-subobjects and

- the pullback of an arrow in $\mathscr{M}$ along an arbitrary map in $\mathscr{A}$ exists and lies again in $\mathscr{M}$. 
The category $\operatorname{Par}^{\mathrm{op}} \mathscr{A}=\operatorname{Par}_{\mathscr{M}}^{\mathrm{op}} \mathscr{A}$ of co- $\mathscr{M}$-partial maps is defined to have

- the same objects as $\mathscr{A}$;

- morphism in $\operatorname{Par}^{\mathrm{op}} \mathscr{A}$ are equivalence classes of spans in $\mathscr{A}$ of the type $\bullet \stackrel{\mathscr{A}}{\longleftarrow} \stackrel{\mathscr{M}}{\longrightarrow} \bullet$ i.e., where the second leg is required to lie in $\mathscr{M}$;

- composition in $\operatorname{Par}^{\mathrm{op}} \mathscr{A}$ is that of spans, i.e., by pullback.

We define two wide subcategories $E, E^{\vee} \subset \operatorname{Par}^{\mathrm{op}} \mathscr{A}$ to consist of the spans of the type

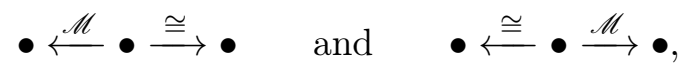

respectively. With the notation of Section 3.1 the Monos are the spans of type $\bullet \stackrel{\mathscr{E}}{\leftarrow} \stackrel{\mathscr{M}}{\longrightarrow} \bullet$. The set Reg of regular morphisms consists of those morphisms in $\operatorname{Par}^{\mathrm{op}} \mathscr{A}$ which are totally defined, i.e., the spans of type $\bullet \stackrel{\mathscr{A}}{\longleftarrow} \stackrel{\cong}{\rightrightarrows} \bullet$; hence we have $\operatorname{Reg} \cong \mathscr{A}^{\mathrm{op}}$.

Lemma 4.2.1. The datum $\mathbb{B}:=\left(\operatorname{Par}^{\mathrm{op}} \mathscr{A}, E, E^{\vee}\right)$ is a diagonalizable DK-triple.

Proof. The proof is straightforward and left to the reader.

The regular Monos in $\operatorname{Par}^{\text {op }} \mathscr{A}$ are the spans of the type $\bullet \stackrel{\mathscr{E}}{\leftarrow} \cong \stackrel{\rightrightarrows}{\bullet}$; they form a subcategory equivalent to $\mathscr{E}^{\mathrm{op}}$. Hence Remark 3.2.2 says that the pointed category $N_{0}(\mathbb{B})$ constructed in Construction 3.2.1 is just the free pointed category $\mathscr{E}_{+}^{\text {op }}$ on $M \cap \operatorname{Reg} \cong \mathscr{E}_{\mathrm{op}}$.

The upshot of this discussion is the following corollary of Corollary 3.3.4 taking into account that $N_{0}=\mathscr{E}_{+}^{\mathrm{op}}$ is a free pointed category (see Remark 3.3.5) and that the DK-triple $\mathbb{B}$ is diagonalizable (see Remark 3.3.8).

Corollary 4.2.2. Let $\mathscr{A}$ and $(\mathscr{E}, \mathscr{M})$ be as above. The DK-triple $\left(\operatorname{Par}_{\mathscr{M}}^{\mathrm{op}} \mathscr{A}, E, E^{\vee}\right)$ induces a natural equivalence

$$
\operatorname{Fun}\left(\operatorname{Par}_{\mathscr{M}}^{\mathrm{op}} \mathscr{A}, \mathcal{A}\right) \stackrel{\simeq}{\longrightarrow} \operatorname{Fun}\left(\mathscr{E}^{\mathrm{op}}, \mathcal{A}\right)
$$

for each weakly idempotent complete preadditive $\infty$-category $\mathcal{A}$.

The prototypical example of Corollary 4.2.2 comes from the category Fin of finite sets, equipped with its canonical surjective-injective factorization system (Surj, Inj); in this case $\operatorname{Par}^{\mathrm{op}} \mathbf{F i n}$ is precisely Segal's category $\Gamma=\mathbf{F i n}_{\star}^{\mathrm{op}}$ [Seg74], hence we get a natural equivalence

$$
\operatorname{Fun}(\Gamma, \mathcal{A}) \stackrel{\simeq}{\longrightarrow} \operatorname{Fun}\left(\operatorname{Surj}^{\text {op }}, \mathcal{A}\right)
$$

or, after dualizing (see Remark 3.3.2),

$$
\operatorname{Fun}\left(\Gamma^{\mathrm{op}}, \mathcal{A}\right) \stackrel{\simeq}{\longrightarrow} \operatorname{Fun}(\operatorname{Surj}, \mathcal{A}),
$$

for all weakly idempotent complete preadditive $\infty$-categories $\mathcal{A}$.

We refer the reader to [LS15, Section 7] for many more examples in this spirit.

Remark 4.2.3. Up to minor differences 14 , the pairs $\left(\operatorname{Par}_{\mathscr{M}} \mathscr{A}, \mathscr{E}\right)$ arising from a factorization system $(\mathscr{E}, \mathscr{M})$ as above are the conjugate pairs $(\mathcal{B}, \mathcal{A})$ of Helmstutler [Hel14]. For the convenience of the reader we provide a table translating Helmstutler's notation to the one of Lack and Street (which we are using in this section):

\begin{tabular}{c|c|c|c|c} 
Lack and Street & $\mathscr{A}$ & $\mathscr{M}$ & $\mathscr{E}$ & $\operatorname{Par}_{\mathscr{M}} \mathscr{A}$ \\
\hline Helmstutler & $\mathcal{U}$ & $\mathcal{J}$ & $\mathcal{A}$ & $\mathcal{B}$
\end{tabular}

\footnotetext{
14) For instance, Helmstutler's $\mathscr{M}$ is not required to contain all isomorphisms. Unlike Lack and Street (hence us) he does however require the pullback of an arrow in $\mathscr{E}$ along an arrow in $\mathscr{M}$ to lie in $\mathscr{E}$ again; this amounts to saying that the set $M$ of Monos is closed under composition.
} 
Helmstutler calls the arrows in $\mathscr{A} \subset$ Par $\mathscr{M} \mathscr{A}$ regular (because they are totally, and not just $\mathscr{M}$-partially, defined) and the other arrows in $\operatorname{Par}_{\mathscr{M}} \mathscr{A}$ singular; this matches our use of those words. Moreover, he constructs a bimodule $\mathcal{U}_{+}: \mathcal{A}^{\mathrm{op}} \times \mathcal{B} \longrightarrow$ Set $_{\star}$ (which is precisely our bimodule $R_{0}$ from Construction 3.2.4 and proves [Hel14, Theorem 6.2] that it induces, for each left proper stable model category $\mathcal{C}$, a Quillen equivalence $\left[\mathcal{B}^{\mathrm{op}}, \mathrm{C}\right] \rightleftarrows\left[\mathcal{A}^{\mathrm{op}}, \mathrm{C}\right]$ (left adjoint on top). Corollary 4.2.2 is our version of this result, where we replace a Quillen equivalence of model categories by an equivalence of $\infty$-categories. Note that the self-duality inherent to our $\infty$-categorical approach (see Remark 3.3.2) fixes the asymmetry problem addressed by Helmstutler in the note at the end of Section 6 in [Hel14].

\section{The proof}

\subsection{Cofinality lemmas}

In order to get a better understanding of the Kan extensions appearing in Theorem 3.3.1 we use cofinality arguments to simplify the relevant pointwise formulas.

Construction 5.1.1. Fix an element $n \in N$. Consider the category

$$
X_{n}:=E^{\vee}([n]) \sqcup_{E_{\nsucceq}^{\vee}([n])} E_{\nsucceq}^{\vee}([n])^{\triangleright},
$$

equipped with the functor $X_{n} \rightarrow B_{+/ n}$ given by sending each $b \in E^{\vee}([n])$ to the composition $b \longmapsto[n] \rightarrow n$ (which is the zero map for all $\left.b \in E_{\nsucceq}^{\vee}([n])\right)$ and the cone point of $E_{\nsucceq}^{\vee}([n])^{\triangleright}$ to $0 \rightarrow n$. Since $E_{\nsucceq}^{\vee}([n])$ is (equivalent to) a poset, the same is true for $X_{n}$; the latter poset arises from $E^{\vee}([n])$ by adding one new element which is bigger than all elements of $E^{\vee}([n])$ except its terminal object Id: $[n] \longmapsto[n]$.

Fix an element $b \in B$. Denote by $Y_{b} \subset\left(N_{0}\right)_{b}$ the (non-full) subcategory defined as follows:

- objects are the maps $b \rightarrow \underline{b}^{\prime}$ corresponding to $E$ pis $b \rightarrow b^{\prime}$ in $B$ (recall that $\underline{b^{\prime}} \in N$ denotes the object corresponding to $b^{\prime} \in B$ ) and the unique map $b \rightarrow 0$.

- the only morphisms are isomorphisms under $b$ and the zero morphisms $\underline{b^{\prime}} \rightarrow 0$.

Observe that $Y_{b}$ is equivalent to the right cone

$$
\left\{b \rightarrow b^{\prime}\right\}^{\triangleright}
$$

on the discrete set $\left\{b \rightarrow b^{\prime}\right\}$ containing some choice of representatives for the isomorphism classes of $E$ pis out of $b$; the cone point corresponds to the object $b \rightarrow 0$ of $Y_{b}$.

Lemma 5.1.2. For each $n \in N$, the inclusion $X_{n} \longleftrightarrow B_{+/ n}$ is homotopy terminal.

Before we go into the rather technical proof of Lemma 5.1.2, we state the direct following corollary which is what we will use going forward.

Corollary 5.1.3. Let $\mathcal{P}$ be a pointed $\infty$-category and $\mathcal{X}: B_{+} \rightarrow \mathcal{P}$ a pointed diagram. Then a left Kan extension $\mathcal{X}^{1}$ of $\mathcal{X}$ along the inclusion $B_{+} \hookrightarrow V$ exists if and only if for each $n \in N$ the total cofiber of the diagram

$$
E^{\vee}([n]) \longrightarrow B_{+} \stackrel{\mathcal{X}}{\longrightarrow} \mathcal{A}
$$

exists in $\mathcal{A}$. If it exists, this left Kan extension $\mathcal{X}^{1}$ is characterized pointwise at $n \in N$ by the fact that it extends the diagram

$$
X_{a} \longrightarrow B_{+/ n} \longrightarrow B_{+} \stackrel{\mathcal{X}}{\longrightarrow} \mathcal{A}
$$

to a colimit cone in $\mathcal{A}$ with colimit $\mathcal{X}^{1}(n)$. 
Remark 5.1.4. If the colimit of the diagram

$$
E_{\nsucceq}^{\vee}([n]) \longrightarrow B_{+} \stackrel{\mathcal{X}}{\longrightarrow} \mathcal{A}
$$

exists for each $n \in N$ then we can characterize the left Kan extension as in Corollary 5.1.3 by the fact that it induces cofiber sequences

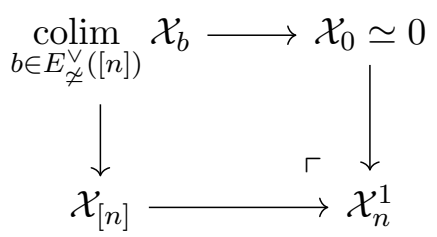

We will show in Proposition 5.2.1 that this colimit always exists when $\mathcal{A}$ is weakly idempotent complete additive.

Proof (of Lemma 5.1.2). Fix $n \in N$ and let us abbreviate $X:=X_{n}$ to avoid proliferating subscripts. For each $b \in B$ and each arrow $u: b \rightarrow n$ in $V$, the undercategory $X_{u /}$ can be described explicitly as follows:

- objects are factorizations

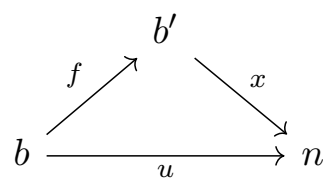

of $u$ in $V$, where the arrow $x: b^{\prime} \rightarrow n$ is required to lie in $X$;

- a morphism $(x, f) \rightarrow\left(x^{\prime}, f^{\prime}\right)$ between such factorizations is simply an arrow $x \rightarrow x^{\prime}$ in $X$ compatible with $f$ and $f^{\prime}$.

Observe that $X_{u}$ is a poset (because $X$ is). To prove that $X \hookrightarrow B_{+/ n}$ is homotopy initial we have to show that all these categories of factorizations are weakly contractible. We distinguish two cases:

- Assume that $u: b \rightarrow n$ is a non-zero. Then the only factorization of $u$ through an object of $X$ is the tautological factorization $u: b \stackrel{[u]}{\longrightarrow}[n] \stackrel{!}{\rightarrow} n$ hence the category $X_{u}$ is a singleton.

- Assume that $u: b \stackrel{0}{\rightarrow} u$ is the zero map. In this case there are three types of factorizations:

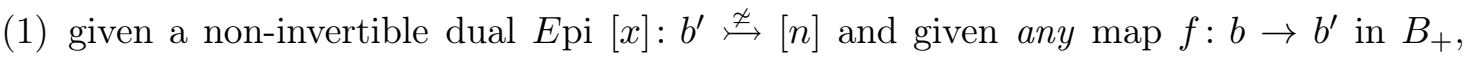
there is a factorization $0: b \stackrel{f}{\rightarrow} b^{\prime} \stackrel{x}{\rightarrow} n$;

(2) for each singular map $s: b \rightarrow[n]$ in $B_{+}$, there is a factorization $0: b \stackrel{s}{\rightarrow}[n] \stackrel{!}{\rightarrow} n$;

(3) there is the zero factorization $0: b \rightarrow 0 \rightarrow n$.

Denote by $(x, f),(!, s)$ and 0 the objects of $X_{u /}$ corresponding to the factorizations of type (1), (2) and (3), respectively. Denote by $Z \subset X_{u}$ the subposet consisting of the objects $(x, f)$ of the first. For each singular map $s: b \rightarrow[n]$, denote by $Z_{s} \subset Z$ the subposet consisting of those $(x, f)$, where the composite $b \stackrel{f}{\rightarrow} b^{\prime} \stackrel{[x]}{\longrightarrow}[n]$ is equal to $s: b \rightarrow[n]$. We now describe the morphisms in the category $X_{u /}$.

- For each factorization $(x, f)$ with $s:=[x] \circ f$ (as maps $b \rightarrow[n]$ ), we have a unique map $(x, f) \rightarrow(!, s)$. There are no other maps between factorizations of types (1) and (2). In other words, the subposet $Z_{s} \cup\{(!, s)\} \subset X_{u}$ is a (right) cone on $Z_{s}$ with maximum $(!, s)$

- There are no maps between factorizations of types (2) and (3) (because there are no maps between $[n] \rightarrow n$ and $0 \rightarrow n$ in $X$ )

- For each factorization $(x, f)$, we have a unique map $(x, f) \rightarrow 0$. There are no other maps between factorizations of types (1) and (3). In other words, the subposet $Z \cup$ $\{0\} \subset X_{u /}$ is a (right) cone on $Z$ with maximum 0 . 
It follows that we have the following pushout of simplicial sets:

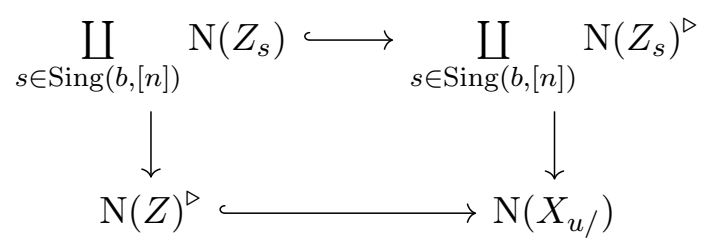

which is a (Kan) homotopy pushout because the top horizontal map is a monomorphism. By (T1), each singular arrow $s: b \rightarrow[n]$ admits a unique (up to unique isomorphism) factorization $s: b \rightarrow b^{\prime} \stackrel{\sharp}{\sharp}[n]$, where $b \rightarrow b^{\prime}$ is regular and $b^{\prime} \stackrel{\sharp}{\sharp}[n]$ is a non-invertible dual $E$ pi; viewed as a factorization of $0: b \rightarrow n$ it is an initial object of the category $Z_{s}$, which is hence contractible. Therefore the top horizontal map in the square (5.1) is a (Kan) weak equivalence, hence also the bottom horizontal map; this concludes the proof because $\mathrm{N}(Z)^{\triangleright}$ is contractible.

Lemma 5.1.5. For each $b \in B$, the inclusion $Y_{b} \longleftrightarrow\left(N_{0}\right)_{b /}$ is homotopy initial.

Proof. Fix $b \in B$ and abbreviate $Y:=Y_{b}$. Similarly to the proof of Lemma 5.1.2, we have to show that, for each $n \in N_{0}$ and each map $u: b \rightarrow n$, the groupoid $Y_{/ u}$ of factorizations

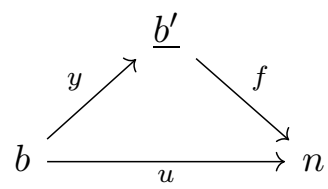

(with $y \in Y$ ) is weakly contractible. Again, we distinguish two cases:

- If the arrow $u: b \rightarrow n$ is non-zero then factorizations (5.2) are the same as $(M \cap \operatorname{Reg}) \circ E$ factorizations of the corresponding regular map $[u]: b \rightarrow[n]$. By (T1) the groupoid of such factorizations is (equivalent to) a point.

- If the arrow $u$ is the zero then the zero factorization $u: b \rightarrow 0 \rightarrow n$ is a terminal object of the category $Y_{/ u}$, which is hence contractible.

Corollary 5.1.6. Let $\mathcal{A}$ be a pointed $\infty$-category with finite products. Every pointed diagram $\overline{\mathcal{X}}: N_{0} \rightarrow \mathcal{A}$ admits a right Kan extension $\mathcal{X}: V \rightarrow \mathcal{A}$ along the inclusion $N_{0} \hookrightarrow V$. Moreover, this right Kan extension is characterized pointwise by the product cones

$$
\mathcal{X}_{b} \stackrel{\simeq}{\longrightarrow} \prod_{b \rightarrow n} \overline{\mathcal{X}}_{n}
$$

indexed by equivalence classes of Epis out of $b$.

Proof. Let $\overline{\mathcal{X}}: N_{0} \rightarrow \mathcal{A}$ be a pointed diagram and fix an objects $b \in B$. By Lemma 5.1.5 we can compute the pointwise right Kan extension $\mathcal{X}$ of $\overline{\mathcal{X}}$ along $N_{0} \hookrightarrow V$ at $b$ as the limit

$$
\mathcal{X}_{b} \stackrel{\simeq}{\longrightarrow} \lim \left(\left(N_{0}\right)_{b /} \rightarrow N_{0} \stackrel{\overline{\mathcal{X}}}{\longrightarrow} \mathcal{A}\right) \stackrel{\simeq}{\longrightarrow} \lim \left(Y_{b} \rightarrow N_{0} \stackrel{\overline{\mathcal{X}}}{\longrightarrow} \mathcal{A}\right) \simeq \lim \left(\{b \rightarrow n\}^{\triangleright} \rightarrow N_{0} \stackrel{\overline{\mathcal{X}}}{\longrightarrow} \mathcal{A}\right) .
$$

This limit formula is the same as the product formula (5.3) because the value of $\overline{\mathcal{X}}$ on the cone point of $\{b \rightarrow n\}^{\triangleright}$ is $\overline{\mathcal{X}}_{0} \simeq 0$.

\subsection{Inductive construction in the reduced case}

Throughout this section we assume that the $\mathrm{DK}$-triple $\mathbb{B}=\left(B, E, E^{\vee}\right)$ is reduced, i.e., that $B=E^{\vee} \circ E$ and hence $N_{0}=B_{+}^{\simeq}$. By applying Construction 3.2.4 to the reduced DK-triple $\mathbb{B}$ and to its dual $\mathbb{B}^{\text {op }}$, we obtain two categories

$$
V=V(\mathbb{B}):=\left(\begin{array}{cc}
N_{0}^{1} & R_{0} \\
0 & B_{+}
\end{array}\right) \quad \text { and } \quad V^{\vee}:=V\left(\mathbb{B}^{\mathrm{op}}\right)^{\mathrm{op}}=\left(\begin{array}{cc}
B_{+} & M_{0} \\
0 & N_{0}^{0}
\end{array}\right)
$$


where $N_{0}^{0}$ and $N_{0}^{1}$ are both just (a copy of) $N_{0}$, decorated with superscripts 0 and 1 to avoid confusing them. For every $n \in N$ we denote by $n_{0}$ its copy in $N^{0} \subset V^{\vee}$ and by $n_{1}$ its copy in $N^{1} \subset V$. Furthemore, we denote by $V_{\leq n} \subset V$ the full subcategories spanned by $B_{+}$and by all the objects $n_{1}^{\prime}$ with $n^{\prime} \leq n$; similarly, $V_{\leq n}^{\vee} \subset V^{\vee}$ is the full subcategory which contains $B_{+}$and all the objects $n_{0}^{\prime}$ with $n^{\prime} \leq n$.

Proposition 5.2.1. Let $\mathcal{A}$ be a weakly idempotent complete additive $\infty$-category $\mathcal{A}$ and let $\mathcal{X}: B_{+} \rightarrow \mathcal{A}$ be a pointed functor. Then there exist functors

$$
\mathcal{X}^{0}: V^{\vee} \rightarrow \mathcal{A} \quad \text { and } \quad \mathcal{X}^{1}: V \rightarrow \mathcal{A}
$$

which are right and left Kan extension of $\mathcal{X}$, respectively. Moreover the functors $\mathcal{X}^{0}$ and $\mathcal{X}^{1}$ are a left Kan extension and a right Kan extension of their restriction to $N_{0}^{0}$ and $N_{0}^{1}$, respectively.

Remark 5.2.2. By Corollary 5.1.6, the "moreover" part of Proposition 5.2.1 is saying that for each $b \in B$ the diagrams $\mathcal{X}^{0}$ and $\mathcal{X}^{1}$ induce direct sum decompositions

$$
\coprod_{n \smile b} \mathcal{X}^{0}(n) \stackrel{\simeq}{\longrightarrow} \mathcal{X}^{0}(b)=\mathcal{X}_{b} \quad \text { and } \quad \mathcal{X}_{b}=\mathcal{X}^{1}(b) \stackrel{\simeq}{\longrightarrow} \prod_{b \rightarrow n} \mathcal{X}^{1}(n)
$$

where the coproduct/product is indexed over equivalence classes of dual Epis into $b$ and Epis out of $b$, respectively.

Remark 5.2.3. It follows from the universal property of the coproduct that each dual $E$ pi $b^{\prime} \longmapsto b$ induces a commutative square

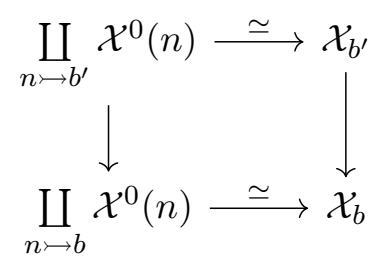

where the left vertical map is the inclusion of those summands that are labeled by a dual $E$ pi which factors through $b^{\prime} \longmapsto b$. Similarly each $E$ pi $b \rightarrow b^{\prime}$ induces projection onto those factors of the decomposition $\mathcal{X}_{b} \simeq \prod_{b \rightarrow n} \mathcal{X}^{1}(n)$ that are indexed by Epis which factor through $b \rightarrow b^{\prime}$.

Proof. For each $n \in N$ we prove:

(1) A right Kan extension $\mathcal{X}_{\leq n}^{0}$ of $\mathcal{X}$ along $B_{+} \hookrightarrow V_{\leq n}^{\vee}$ exists.

(2) A left Kan extension $\mathcal{X}_{\leq n}^{1}$ of $\mathcal{X}$ along $B_{+} \hookrightarrow V_{\leq n}$ exists.

(3) Each choice of such Kan extensions $\mathcal{X}_{\leq n}^{0}$ and $\mathcal{X}_{\leq n}^{1}$ induces, for each $b \leq[n]$, direct sum decompositions as in (5.4); moreover, the composition

$$
\mathcal{X}_{\leq n}^{0}\left(n_{0}\right) \longrightarrow \mathcal{X}_{[n]} \longrightarrow \mathcal{X}_{\leq n}^{1}\left(n_{1}\right)
$$

is an equivalence in $\mathcal{A}$.

By induction on the number $\left|\pi_{0} E^{\vee}(n)\right|=\left|\pi_{0} E(n)\right|$ we may assume that we have proved (1), (2) and (3) for all objects of $N$ which are strictly smaller than $n$. Choose a right Kan extension $\mathcal{X}_{<n}^{0}: V_{<n}^{\vee} \rightarrow \mathcal{A}$ and a left Kan extension $\mathcal{X}_{<n}^{1}: V_{<n} \rightarrow \mathcal{A}$ of $\mathcal{X}: B_{+} \rightarrow \mathcal{A}$ (they exist pointwise by assumption). By assumption, $\mathcal{X}_{<n}^{0}$ induces coproduct decompositions $\coprod_{n^{\prime} \rightarrow b} \mathcal{X}_{<n}^{0}\left(n^{\prime}\right) \stackrel{\simeq}{\rightarrow} \mathcal{X}_{b}$ for all $b<[n]$. Since all dual Epis induce compatible inclusions of summands (see Remark 5.2.3), the diagram $\mathcal{X}^{0}$ provides an identification

$$
\coprod_{n^{\prime} \varkappa_{[n]}} \mathcal{X}_{<n}^{0}\left(n^{\prime}\right) \simeq \operatorname{colim}_{b \in E_{\nsucceq}^{\forall}([n])} \mathcal{X}_{b}
$$


where the coproduct is indexed over equivalence classes of non-invertible dual Epis; moreover, this identification (5.5) is compatible with the respective structure maps to $\mathcal{X}_{[n]}$. By applying the dual argument to $\mathcal{X}_{<n}^{1}: V_{<n} \rightarrow \mathcal{A}$ we obtain an identification

$$
\lim _{b \in E_{\nsucceq}([n])} \mathcal{X}_{b} \simeq \prod_{[n] \varlimsup_{\sharp n^{\prime}}} \mathcal{X}_{<n}^{1}\left(n^{\prime}\right)
$$

again compatible with the structure maps from $\mathcal{X}_{[n]}$. We analyze the two composable maps

$$
\coprod_{n^{\prime} \neq[n]} \mathcal{X}_{<n}^{0}\left(n^{\prime}\right) \longrightarrow \mathcal{X}_{[n]} \longrightarrow \prod_{[n] \varkappa_{\rightarrow n^{\prime \prime}}} \mathcal{X}_{<n}^{1}\left(n^{\prime \prime}\right)
$$

and their composite $\Phi$ in terms of the components $\Phi_{n^{\prime \prime}, n^{\prime}}: \mathcal{X}_{<n}^{0}\left(n^{\prime}\right) \rightarrow \mathcal{X}_{[n]} \rightarrow \mathcal{X}_{<n}^{1}\left(n^{\prime \prime}\right)$. We have the commutative diagram in $V_{<n}^{\vee} \sqcup_{B_{+}} V_{<n}$

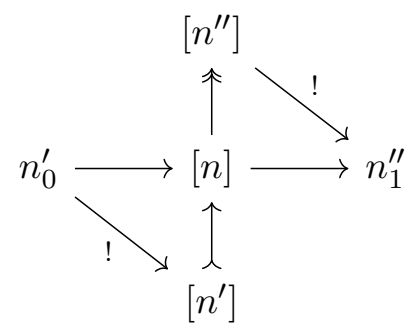

where the vertical morphisms are the dual $E$ pi $\left[n^{\prime}\right] \longmapsto[n]$ and the $E$ pi $[n] \rightarrow\left[n^{\prime}\right]$; their composition is - by definition - the map $\left\langle\left[n^{\prime \prime}\right] ;\left[n^{\prime}\right]\right\rangle_{[n]}$. Therefore, the map $\Phi_{n^{\prime \prime}, n^{\prime}}$ is equivalent to the composition

$$
\Phi_{n^{\prime \prime}, n^{\prime}}: \mathcal{X}_{<n}^{0}\left(n_{0}^{\prime}\right) \longrightarrow \mathcal{X}_{\left[n^{\prime}\right]} \stackrel{\mathcal{X}\left(\left\langle\left[n^{\prime \prime}\right] ;\left[n^{\prime}\right]\right\rangle_{[n]}\right)}{\longrightarrow} \mathcal{X}_{\left[n^{\prime \prime}\right]} \longrightarrow \mathcal{X}_{<n}^{1}\left(n_{1}^{\prime \prime}\right)
$$

It follows that:

- If $\left\langle\left[n^{\prime \prime}\right] ;\left[n^{\prime}\right]\right\rangle_{[n]}$ is an isomorphism in $B_{+}$(without loss of generality, the identity) then $\Phi_{n^{\prime \prime}, n^{\prime}}$ is an equivalence by the induction hypothesis (3).

- If $\left\langle\left[n^{\prime \prime}\right] ;\left[n^{\prime}\right]\right\rangle_{[n]}$ is not an isomorphism in $B_{+}$then it must be either singular or cosingular. If it is singular then the composition $\left[n^{\prime}\right] \rightarrow\left[n^{\prime \prime}\right] \rightarrow n_{1}^{\prime \prime}$ factors through $0 \in V$; if it is cosingular then the composition $n_{0}^{\prime} \rightarrow\left[n^{\prime}\right] \rightarrow\left[n^{\prime \prime}\right]$ factors through $0 \in V^{\vee}$; in either case $\Phi_{n^{\prime \prime}, n^{\prime}}$ factors through $\mathcal{X}_{0} \simeq 0$.

Therefore it follows from (T2) that $\left(\Phi_{n^{\prime \prime}, n^{\prime}}\right)$ is an upper triangular matrix with invertible diagonal entries; hence $\Phi$ is invertible because $\mathcal{A}$ is additive (see Lemma 2.4.5). This means that the two composable maps (5.6) are a section-retraction pair. Since $\mathcal{A}$ is weakly idempotent complete, this section-retraction pair admits a complement, i.e., there is an essentially unique diagram

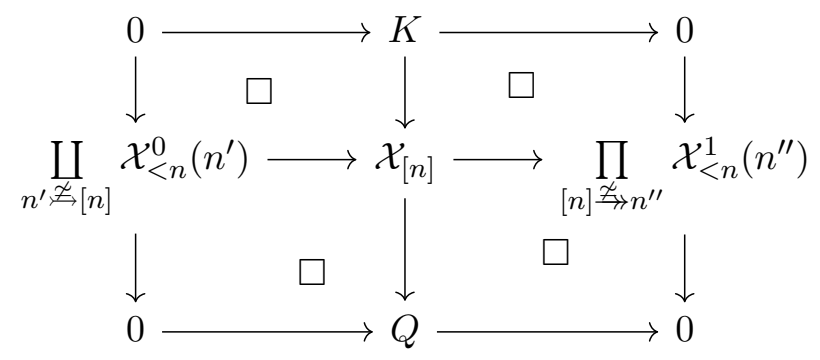

where all squares are biCartesian. By 5.1.3 (or, more precisely, by 5.1.4) and the identification (5.5), we conclude that the pointwise left Kan extension $\mathcal{X}^{1}(n)$ of $\mathcal{X}$ at $n_{1}$ exists and that its value on the structure map !: $[n] \rightarrow n_{1}$ is equivalent to the projection $\mathcal{X}_{[n]} \rightarrow Q$. By the dual argument, we conclude that the pointwise right Kan extension $\mathcal{X}^{0}(n)$ of $\mathcal{X}$ at $n_{0}$ exists and that its value on the structure map $!: n_{0} \rightarrow[n]$ is equivalent to the inclusion $K \rightarrow \mathcal{X}_{[n]}$. To establish 
the inductive step for property (3), note that the diagram (5.7) encodes the required coproduct decompositions

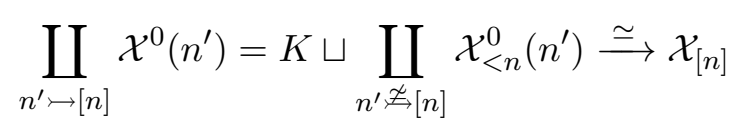

(and similarly the required product decomposition) and the fact that the composition

$$
K \simeq \mathcal{X}^{0}(n) \stackrel{\mathcal{X}^{0}(!)}{\longrightarrow} \mathcal{X}_{[n]} \stackrel{\mathcal{X}^{1}(!)}{\longrightarrow} \mathcal{X}^{1}(n) \simeq Q
$$

is an equivalence.

Remark 5.2.4. If the DK-triple $\mathbb{B}$ is diagonalizable then the matrix $\left(\Phi_{n^{\prime \prime}, n^{\prime}}\right)_{n^{\prime \prime}, n^{\prime}}$ is actually a diagonal matrix. Hence to invert it, we do not need $\mathcal{A}$ to be additive but only preadditive.

Remark 5.2.5. From the proof of Proposition 5.2.1 we can extract more detailed information. For each $n \in N$, the extensions $\mathcal{X}^{0}$ and $\mathcal{X}^{1}$ encode two complementary section-retraction pairs

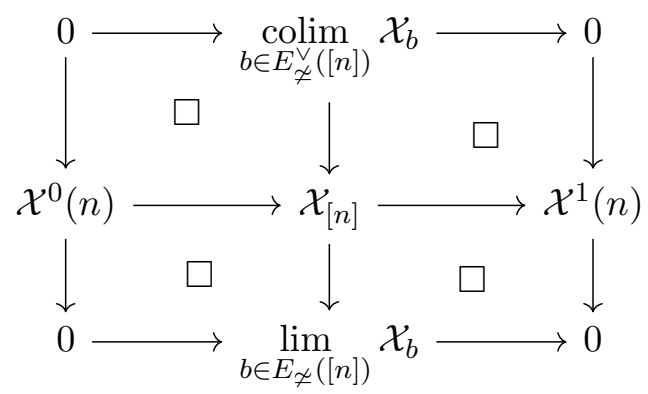

(in particular, the indicated limits/colimits exist).

\subsection{Proof of the main theorem}

Proof (of Theorem 3.3.1). We first prove (a), (b), and (d) in the case where the DK-triple $\left(B, E, E^{\vee}\right)$ is reduced. In this case, we have the following ingredients:

- Corollary 5.1.6 guarantees that the right Kan extension functor $\operatorname{RKE:~} \operatorname{Fun}^{0}\left(N_{0}, \mathcal{A}\right) \rightarrow$ $\operatorname{Fun}^{0}(V, \mathcal{A})$ exists. Moreover, the explicit formula (5.3) implies that for any natural transformation $\alpha: \overline{\mathcal{X}}^{\prime} \rightarrow \overline{\mathcal{X}}$ of pointed diagrams $N_{0} \rightarrow \mathcal{A}$, the component $\alpha_{n}: \overline{\mathcal{X}}_{n}^{\prime} \rightarrow \overline{\mathcal{X}}_{n}$ at $n \in N$ is a factor of the corresponding right Kan extended transformation (with the notation as in 5.1.6)

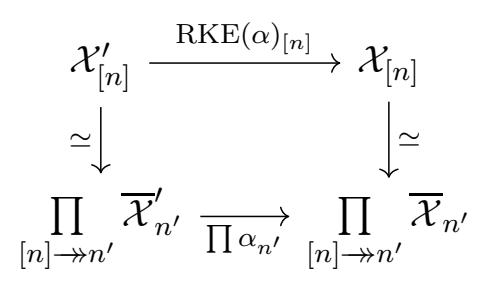

at $[n] \in B$; hence it follows from 2.2.6 that the composition

$$
\operatorname{Fun}^{0}\left(N_{0}, \mathcal{A}\right) \stackrel{\operatorname{RKE}}{\longrightarrow} \operatorname{Fun}^{0}(V, \mathcal{A}) \stackrel{\operatorname{Res}}{\longrightarrow} \operatorname{Fun}^{0}\left(B_{+}, \mathcal{A}\right)
$$

is conservative, i.e., reflects equivalences.

- Proposition 5.2.1 states in particular - if we focus only on the statements about $V$ and not about $V^{\vee}$ - that

- the left Kan extension functor $\operatorname{LKE}: \operatorname{Fun}^{0}\left(B_{+}, \mathcal{A}\right) \rightarrow \operatorname{Fun}^{0}(V, \mathcal{A})$ exists and

- on the image of this functor LKE, the unit $\operatorname{Id}_{\operatorname{Fun}^{0}(V, \mathcal{A})} \rightarrow$ RKE $\circ$ Res of the adjunction

$$
\text { Res: } \operatorname{Fun}^{0}(V, \mathcal{A}) \rightleftarrows \operatorname{Fun}^{0}\left(N_{0}, \mathcal{A}\right): \operatorname{RKE}
$$

is an equivalence. 
Since left Kan extension along the fully faithful functor $B_{+} \hookrightarrow V$ is fully faithful, the unit $\operatorname{Id}_{\mathrm{Fun}^{0}\left(B_{+}, \mathcal{A}\right)} \rightarrow$ Res $\circ$ LKE is an equivalence. We conclude that the unit

$$
\operatorname{Id}_{\mathrm{Fun}^{0}\left(B_{+}, \mathcal{A}\right)} \longrightarrow \operatorname{Res} \circ \mathrm{LKE}=\operatorname{Res} \circ \operatorname{Id}_{\mathrm{Fun}^{0}(V, \mathcal{A})} \circ \mathrm{LKE} \longrightarrow \operatorname{Res} \circ \mathrm{RKE} \circ \operatorname{Res} \circ \mathrm{LKE}
$$

of the composite adjunction 3.2 is also an equivalence.

This already proves (a) assertion (b) follows from the general fact about adjunctions that if the right adjoint is conserative and the unit is an equivalence then the whole adjunction is an adjoint equivalence. Assertion (d) is spelled out in Remark 5.2.5 since $\overline{\mathcal{X}}_{n}$ is by definition equivalent to $\mathcal{X}^{1}(n)$.

To prove (a), (b) and (d) when $\mathbb{B}$ is not necessarily reduced, we make the following key observation:

- the criterion for constructing and detecting left Kan extension along $B_{+} \hookrightarrow V$ (Corollary 5.1.3 and the criterion for constructing and detecting right Kan extension along $N_{0} \hookrightarrow V$ Corollary 5.1.6) both only depend on the values of a diagram on the dual $E$ pis $E^{\vee}$ and on the Epis $E$.

Therefore we can reduce to the reduced case (no pun intended) by replacing the original DK-triple with the reduced DK-triple

$$
\overline{\mathrm{B}}:=\left(E^{\vee} \circ E, E, E^{\vee}\right) .
$$

To prove (c), note that the right Kan extension RKE: $\operatorname{Fun}^{0}\left(N_{0}, \mathcal{A}\right) \longrightarrow \operatorname{Fun}^{0}(V, \mathcal{A})$ is natural in $\mathcal{A}$ with respect to all functors which preserve the relevant pointwise limits; since all these pointwise limits are just products, this is true for every additive functor.

\section{Comparison with...}

\section{1 $\quad$...the setting of Lack and Street}

We provide a short dictionary/comparison between our setup described in Section 3.1 and Section 3.2 and the setting of Lack and Street [LS15, Section 2]. Unless stated otherwise, references in this section refer to their revised arXiv paper [LS14], not the published one [LS15] (see also the corrigendum [LS20]); we freely use the notation of [LS14, Section 2].

Their category $\mathscr{P}$ is the dual of our category $B$. Under this duality we have the following table of correspondence:

\begin{tabular}{c|c|c|c|c|c|c|c} 
Lack and Street & $\mathscr{P}$ & $\mathscr{M}$ & $\mathscr{M}^{*}$ & $\mathscr{R}$ & $\mathscr{D}$ & $\mathscr{S}$ & $\left\{u \mid s_{u} \in \mathscr{R}\right\}$ \\
\hline our setup & $B$ & $E$ & $E^{\vee}$ & $M \cap \operatorname{Reg}$ & $N$ & $M$ & $\operatorname{Reg}$
\end{tabular}

Lack and Street take as part of the data an isomorphism $(-)^{*}: \mathscr{M}^{\text {op }} \cong \mathscr{M}^{*}$ (which in our language would be written as $(-)^{\vee}: E^{\mathrm{op}} \cong E^{\vee}$ ) which is the identity on objects and satisfies $m^{*} \circ m=$ Id for all arrows $m$ in $\mathscr{M}$. Their Assumption 2.5 translates to the fact that the set $\pi_{0} E(b)$ is finite for each $b \in B$; Assumption 2.6 is saying that for each $b \in B$ there exists a linear order on $\pi_{0} E(b)$ such that the matrix $\left\langle-;(-)^{\vee}\right\rangle_{b}: \pi_{0} E(b) \times \pi_{0} E(b) \rightarrow \pi_{0} \operatorname{Ar} B$ has only singular entries below the diagonal. In our setup, (T2) replaces all these ingredients and repackages them as a property which more directly reflects the final use: what we ultimately want to exploit is that certain unipotent upper triangular matrices (5.6) induced from the matrices $\langle-;-\rangle_{b}$ can be inverted in any additive $\infty$-category. Note that while Lack and Street require the matrix entries below the diagonal to be singular, it suffices for our purposes if they are non-invertible.

Furthermore:

- Their Assumption 2.1 and Assumption 2.4 correspond precisely to our axioms (T1) and (T3), respectively.

- Their Assumption 2.2 translates to our axiom (T4) 
- Their Assumption 2.3 translates to $(M \cap \operatorname{Reg}) \circ E_{\nsucceq}^{\vee} \subset B \backslash(M \cap \operatorname{Reg})$ and is, a priori, weaker than our axiom (T5). However, they use Assumption 2.3 (in the presence of the other assumptions) to prove Proposition 2.10(b) which states that if two composable arrows $v, u$ satisfy $s_{v} \notin \mathscr{R}$ and $u \in \mathscr{S}$, then also $s_{v u} \notin \mathscr{R}$. This statement translates to $M \circ$ Sing $\subseteq$ Sing, which is precisely (T5).

The preceding discussion proves:

Corollary 6.1.1. Let $\mathscr{P}, \mathscr{M}, \mathscr{M}^{*}$ and $\mathscr{D}$ be as in [LS14, Section 2]. Then $\mathrm{B}=\left(\mathscr{P}\right.$ op $\left., \mathscr{M}^{\mathrm{op}},\left(\mathscr{M}^{*}\right)^{\mathrm{op}}\right)$ is a DK-triple with associated pointed category $N_{0}(\mathrm{~B})=\frac{\mathscr{D}^{\circ p}}{(0)}$.

The main tool in the proof by Lack and Street is what they call the kernel module [LS15, Section 4]

$$
M: \mathscr{D}^{\mathrm{op}} \times \mathscr{P} \longrightarrow 1 / \text { Set }
$$

(where $1 /$ Set is their notation for the category of pointed sets); it corresponds to our $N_{0^{-}} B_{+^{-}}$ bimodule

$$
R_{0}: B_{+}^{\mathrm{op}} \times N_{0} \longrightarrow \text { Set }_{\star}
$$

which we encode in its upper triangular category $V$. Their main theorem [LS14, Theorem 6.7] [LS15, Theorem 6.8] states that for each idempotent complete additive 1-category $\mathscr{X}$, the kernel module $M$ induces an equivalence

$$
\operatorname{Fun}(\mathscr{P}, \mathscr{X}) \simeq \operatorname{Fun}_{\operatorname{Set}_{\star}}(\mathscr{D}, \mathscr{X})
$$

where Fun Set $_{\star}$ denotes the category Set $_{\star}$-enriched functors. Instead of using Set $_{\star}$-enriched categories (or rather $\mathcal{S}_{\star}$-enriched $\infty$-categories; see also Remark 2.3.7) we chose to work with pointed categories and phrase our main result in terms of pointed functors on $N_{0}=\frac{N}{(0)}$. Therefore Corollary 3.3.6 recovers their result because, for each pointed 1-category $P$ and each Set $_{\star^{-}}$ enriched category $N$, the inclusion $N \hookrightarrow N_{0}$ induces an equivalence of categories $\operatorname{Fun}^{0}\left(N_{0}, P\right) \stackrel{\underset{\leftrightarrows}{\rightrightarrows}}{\rightarrow}$ $\operatorname{Fun}_{\text {Set }_{\star}}(N, P)$ (see Remark 2.3.6).

\section{2 ...Lurie's stable Dold-Kan correspondence}

Let $\mathcal{D}$ be an $\infty$-category with finite colimits and consider the functor

$$
\operatorname{Fun}\left(\Delta^{\mathrm{op}}, \mathcal{D}\right) \longrightarrow \operatorname{Fun}(\mathbb{N}, \mathcal{D})
$$

which sends a simplicial object $\mathcal{X}: \Delta^{\mathrm{op}} \rightarrow \mathcal{D}$ to the filtered object

$$
\hat{\mathcal{X}}: \operatorname{colim} \mathcal{X}_{\leq 0} \longrightarrow \cdots \longrightarrow \operatorname{colim} \mathcal{X}_{\leq n-1} \longrightarrow \operatorname{colim} \mathcal{X}_{\leq n} \longrightarrow \cdots
$$

of its partial colimits $\hat{\mathcal{X}}_{n}:=\operatorname{colim} \mathcal{X}_{\leq n}=\operatorname{colim}\left(\mathcal{X}_{\leq n}: \Delta_{<n}^{\mathrm{op}} \hookrightarrow \Delta^{\mathrm{op}} \stackrel{\mathcal{X}}{\rightarrow} \mathcal{D}\right)$. Lurie's stable DoldKan correspondence [Lur17, Theorem 1.2.4.1] states that the functor (6.1) is an equivalence when the target $\mathcal{D}$ is a stable $\infty$-category. The functor (6.1) lifts the ordinary Dold-Kan correspondence in the following sense: Each filtered object $\hat{\mathcal{X}}$ in a stable $\infty$-category $\mathcal{D}$ gives rise to a connective chain complex

$$
\mathrm{h} \overline{\mathcal{X}}_{0} \longleftarrow \cdots \longleftarrow \mathrm{h} \overline{\mathcal{X}}_{n-1} \longleftarrow \mathrm{h} \overline{\mathcal{X}}_{n} \longleftarrow \cdots
$$

in the homotopy category $\mathrm{h} \mathcal{D}$, with $\overline{\mathcal{X}}_{n}:=\Omega^{n} \operatorname{cof}\left(\hat{\mathcal{X}}_{n-1} \rightarrow \hat{\mathcal{X}}_{n}\right)$. Moreover, there is a commutative diagram
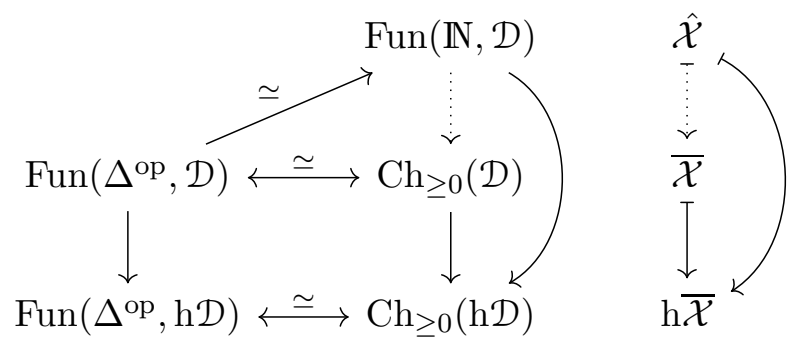
where the top diagonal functor is (6.1) and the lower commutative square is the naturality square of Remark 3.3.7. In particular, the dotted equivalence $\hat{\mathcal{X}} \mapsto \overline{\mathcal{X}}$ exists and functorially lifts the incoherent chain complex (6.2) to a coherent one.

If we only assume that the target $\mathcal{D}$ is weakly idempotent complete additive but not necessarily stable then, even if sufficient colimits exist to define the functor (6.1), it need not be an equivalence anymore; similarly, the dotted functor $\hat{\mathcal{X}} \mapsto \overline{\mathcal{X}}$ (or even $\hat{\mathcal{X}} \mapsto \mathrm{h} \overline{\mathcal{X}}$ ) does not exist in this generality. For instance, in the $\infty$-category of connective spectra the filtered object $0 \rightarrow \mathbb{S} \rightarrow \mathbb{S} \rightarrow \mathbb{S} \rightarrow \ldots$ (which would correspond to the chain complex $0 \leftarrow \mathbb{S}[-1] \leftarrow 0 \leftarrow 0 \leftarrow$ ) does not arise from a simplicial object.

Remark 6.2.1. A systematic study of the relationship between coherent chain complexes and filtered objects in stable $\infty$-categories is part of Stefano Ariotta's Ph.D. thesis Ari. In particular, he directly constructs an equivalence $\operatorname{Fun}(\mathbb{N}, \mathcal{D}) \simeq \mathrm{Ch}_{\geq 0}(\mathcal{D})$ of $\infty$-categories which we expect to agree with the vertical dotted equivalence in (6.3) obtained by combining our result with Lurie's.

\section{Application: measuring Kan extensions}

Let $\mathbb{B}=\left(B, E, E^{\vee}\right)$ be a DK-triple with associated quotient $N_{0}=N_{0}(\mathbb{B})$. Let $\mathcal{X}: B \rightarrow \mathcal{A}$ be a diagram in a weakly idempotent complete additive $\infty$-category $\mathcal{A}$ and let $\overline{\mathcal{X}}: N_{0} \rightarrow \mathcal{A}$ be the pointed functor corresponding to $\mathcal{X}$ under the equivalence of Corollary 3.3.4.

In this section, we set out to answer the following question:

Question 7.0.1. What do the values of the diagram $\bar{X}: N_{0} \rightarrow \mathcal{A}$ tell us about the original diagram $\mathcal{X}: B \rightarrow \mathcal{A}$ ?

The rough answer is that in favorable situations $\overline{\mathcal{X}}$ "measures" how far away $\mathcal{X}$ is from being a Kan extension of its restriction $\mathcal{X}_{<n}$. To make this precise, we make the following definition:

Definition 7.0.2. The DK-triple $\mathbb{B}$ is called monotone if all Monos make objects bigger, i.e., if we have $b^{\prime} \leq b$ whenever there exists a Mono $m: b^{\prime} \rightarrow b$. We say that $\mathbb{B}$ is partially monotone if Monos at least do not make objects smaller, i.e., there are no Monos $b^{\prime} \rightarrow b$ if $b^{\prime}>b$.

Remark 7.0.3. If the partial order $\leq$ on $\pi_{0} B$ is total, then the notions of monotone and partially monotone agree; in general being partially monotone is weaker than being monotone.

Remark 7.0.4. If $\left(\pi_{0} B, \leq\right)$ is the poset $(\mathbb{N}, \leq)$ of natural numbers, then each monotone DK-triple is a generalized Reedy category in the sense of [BM11, Definition 1.1] or [Cis06, Definition 8.1.1] with tautological degree function $\mathrm{Ob} B \rightarrow \pi_{0} B \cong \mathbb{N}$, degree-raising arrows $M$ and degreelowering maps $E$.

Remark 7.0.5. Whether the DK-triple $\left(B, E, E^{\vee}\right)$ is (partially) monotone does not depend on $E^{\vee}$, since both the partial order $\leq$ and the class $M$ of Monos are defined only in terms of the Epis.

Example 7.0.6. - In both the DK-triples $\mathbb{B}_{\min }^{\Delta}$ and $\mathbb{B}_{\max }^{\Delta}$ on $\Delta$ defined in Section 4.1 the partial order $\leq$ on the objects $[n] \in \Delta$ is just the usual comparison of cardinalities; the monos are the injective maps. Hence $\mathbb{B}_{\min }^{\Delta}$ and $\mathbb{B}_{\max }^{\Delta}$ are both monotone.

- Denote by $\mathbb{B}^{\Gamma}$ the DK-triple on $\Gamma$ defined in Section 4.2. It is monotone since the Monos are opposite to the surjective maps in $\mathbf{F i n}_{\star}$ and the order $\leq$ is again just given by comparing cardinalities of finite pointed sets.

Proposition 7.0.7. Let $\mathbb{B}=(B, E, M)$ be a partially monotone DK-triple with associated quotient $N_{0}=N_{0}(\mathrm{~B})$. Fix a diagram $\mathcal{X}: B \rightarrow \mathcal{C}$ in an arbitrary $\infty$-category $\mathcal{C}$ and an object $n \in N$. 
(1) The functor $\mathcal{X}$ is pointwise at $[n] \in B$ a right Kan extension of its restriction to $B_{<[n]}$ if and only if

$$
\left(E_{\nsucceq}([n])\right)^{\triangleleft} \simeq E([n]) \hookrightarrow B \stackrel{\mathcal{X}}{\longrightarrow} \mathcal{C}
$$

is a limit cone in $\mathcal{C}$.

(2) If the $\infty$-category $\mathcal{A}:=\mathcal{C}$ is weakly idempotent complete additive (or preadditive if $\mathbb{B}$ is diagonalizable) then this happens if and only if the corresponding pointed diagram $\overline{\mathcal{X}}: N_{0} \rightarrow \mathcal{A}$ vanishes at $n$, i.e., if and only if $\overline{\mathcal{X}}_{n}$ is a zero object in $\mathcal{A}$.

(3) Assume that $\mathbb{B}$ is monotone. Then $\left.\mathcal{X}\right|_{E(n)}$ is a limit cone if and only if $\mathcal{X}$ is pointwise at $[n] \in B$ a right Kan extension of its restriction to $B_{\nsucceq}[n]$.

Proof. We only prove (1) and (2) the proof of (3) is analogous to (1). The pointwise right Kan extension of $\mathcal{X}_{<[n]}$ at $[n] \in B$ is computed as the limit of the diagram

$$
\lim \left(\left(B_{<[n]}\right)_{[n] /} \longrightarrow B \stackrel{\mathcal{X}}{\longrightarrow} \mathcal{A}\right)
$$

We show that if $\mathbb{B}$ is partially monotone then the canonical inclusion $E_{\nsucceq}([n]) \rightarrow\left(B_{<[n]}\right)_{[n] /}$ is homotopy initial:

- This amounts to showing that for each object $b \in B$ with $b<[n]$ and each arrow $[n] \rightarrow b$, the poset of factorizations

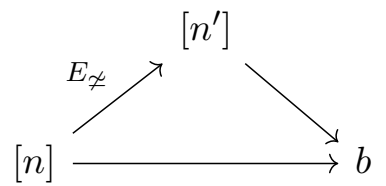

is weakly contractible. The first leg in the unique $(E, M)$-factorization

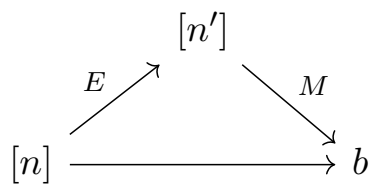

must be non-invertible because otherwise $n^{\prime} \cong n>b$ would contradict the assumption that $\mathbb{B}$ is partially monotone. It follows that the unique factorization (7.2) is of type (7.1) and is therefore a terminal object in the the poset we wish to contract.

It follows that the desired pointwise right Kan extension is computed as the limit

$$
\lim \left(E_{\nsucceq}([n]) \longrightarrow B \stackrel{\mathcal{X}}{\longrightarrow} \mathcal{A}\right)
$$

as required by (1). Statement (2) now follows from Theorem 3.3.1] (d) which states in particular that the canonical map

$$
\mathcal{X}_{[n]} \longrightarrow \lim _{b \in E_{\varkappa}([n])} \mathcal{X}_{b}
$$

is retraction with complement $\overline{\mathcal{X}}_{n}$.

Fix a natural number $k \in \mathbb{N}$. Recall that $\Delta_{\leq k} \subset \Delta$ denotes the full subcategory spanned by the objects $[n]$ with $n \leq k$.

Corollary 7.0.8. A simplicial object $\mathcal{X}: \Delta^{\mathrm{op}} \rightarrow \mathcal{A}$ in a weakly idempotent complete additive $\infty$-category is a left Kan extension of its restriction to $\Delta_{\leq k}^{\mathrm{op}}$ if and only if the corresponding connective chain complex $\overline{\mathcal{X}} \in \mathrm{Ch}_{\geq 0}(\mathcal{A})$ is $k$-truncated, i.e., $\overline{\mathcal{X}}_{n} \simeq 0$ for all $n>k$.

Proof. Apply Proposition 7.0.7 (2) to the DK-triple $\mathbb{B}_{\text {min }}^{\Delta}$ (or, equivalently, to the DK-triple $\mathrm{B}_{\max }^{\Delta}$ ) and dualize. 


\section{References}

[Ari] Stefano Ariotta, Ph.D. thesis, Westfälische Wilhelms-Universität Münster, in preparation.

[BM11] Clemens Berger and Ieke Moerdijk, On an extension of the notion of Reedy category, Math. Z. 269 (2011), no. 3-4, 977-1004. MR 2860274

[Büh10] Theo Bühler, Exact categories, Expo. Math. 28 (2010), no. 1, 1-69. MR 2606234

[CEF15] Thomas Church, Jordan S. Ellenberg, and Benson Farb, FI-modules and stability for representations of symmetric groups, Duke Math. J. 164 (2015), no. 9, 1833-1910. MR 3357185

[Cis06] Denis-Charles Cisinski, Les préfaisceaux comme modèles des types d'homotopie, Astérisque (2006), no. 308, xxiv+390. MR 2294028

[Cis19] Higher categories and homotopical algebra, Cambridge Studies in Advanced Mathematics, vol. 180, Cambridge University Press, Cambridge, 2019. MR 3931682

[Dol58] Albrecht Dold, Homology of symmetric products and other functors of complexes, Ann. of Math. (2) 68 (1958), 54-80. MR 97057

[DP61] Albrecht Dold and Dieter Puppe, Homologie nicht-additiver Funktoren. Anwendungen, Ann. Inst. Fourier Grenoble 11 (1961), 201-312. MR 0150183

[Dug] Daniel Dugger, A primer on homotopy colimits, preprint available at: https://pages.uoregon.edu/ddugger/hocolim.pdf.

[GGN15] David Gepner, Moritz Groth, and Thomas Nikolaus, Universality of multiplicative infinite loop space machines, Algebr. Geom. Topol. 15 (2015), no. 6, 3107-3153. MR 3450758

[Hel14] R. Helmstutler, Conjugate pairs of categories and Quillen equivalent stable model categories of functors, J. Pure Appl. Algebra 218 (2014), no. 7, 1302-1323. MR 3168497

[Joy08] André Joyal, Notes on quasi-categories, Lecture Notes, 2008.

[Kan58] Daniel M. Kan, Functors involving c.s.s. complexes, Trans. Amer. Math. Soc. 87 (1958), 330-346. MR 131873

[LS14] Stephen Lack and Ross Street, Combinatorial categorical equivalences of Dold-Kan type, arXiv e-prints (2014), arXiv:1402.7151v5.

[LS15] Stephen Lack and Ross Street, Combinatorial categorical equivalences of Dold-Kan type, J. Pure Appl. Algebra 219 (2015), no. 10, 4343-4367. MR 3346494

[LS20] - Corrigendum to "Combinatorial categorical equivalences of Dold-Kan type" [J. Pure Appl. Algebra 219 (10) (2015) 4343-4367], J. Pure Appl. Algebra 224 (2020), no. 3, 1364-1366. MR 4009582

[Lur09] Jacob Lurie, Higher topos theory, Annals of Mathematics Studies, vol. 170, Princeton University Press, Princeton, NJ, 2009. MR 2522659

[Lur17] _ Higher algebra, September 2017, Available at the author's homepage.

[Lur18] _ Spectral algebraic geometry (under construction!), February 2018, Available at the author's homepage. 
[ML98] Saunders Mac Lane, Categories for the working mathematician, second ed., Graduate Texts in Mathematics, vol. 5, Springer-Verlag, New York, 1998. MR 1712872

[Pir00] Teimuraz Pirashvili, Dold-Kan type theorem for $\Gamma$-groups, Math. Ann. 318 (2000), no. 2, 277-298. MR 1795563

[Seg74] Graeme Segal, Categories and cohomology theories, Topology 13 (1974), 293-312. MR 0353298

[Sło04] Jolanta Słomińska, Dold-Kan type theorems and Morita equivalences of functor categories, J. Algebra 274 (2004), no. 1, 118-137. MR 2040866

[Sło11] Jolanta Słomińska, Morita equivalences of functor categories and decompositions of functors defined on a category associated to algebras with one-side units, Bull. Pol. Acad. Sci. Math. 59 (2011), no. 1, 33-40. MR 2810970

[TT90] R. W. Thomason and Thomas Trobaugh, Higher algebraic K-theory of schemes and of derived categories, The Grothendieck Festschrift, Vol. III, Progr. Math., vol. 88, Birkhäuser Boston, Boston, MA, 1990, pp. 247-435. MR 1106918 